Article

\title{
Refined 2D and Exact 3D Shell Models for the Free Vibration Analysis of Single- and Double-Walled Carbon Nanotubes
}

\author{
Salvatore Brischetto $^{1, *}$, Francesco Tornabene ${ }^{2, \dagger}$, Nicholas Fantuzzi ${ }^{2, \dagger}$ and \\ Michele Bacciocchi ${ }^{2, \dagger}$ \\ ${ }^{1}$ Department of Mechanical and Aerospace Engineering, Politecnico di Torino, corso Duca degli \\ Abruzzi 24, Torino 10129, Italy \\ 2 Department of Civil, Chemical, Enviromental and Material Engineering, Alma Mater Studiorum, \\ University of Bologna, viale Risorgimento 2, Bologna 40136, Italy; \\ E-Mails: francesco.tornabene@unibo.it (F.T.); nicholas.fantuzzi@unibo.it (N.F.); \\ michele.bacciocchi@unibo.it (M.B.) \\ $\dagger$ These authors contributed equally to this work. \\ * Author to whom correspondence should be addressed; E-Mail: salvatore.brischetto@polito.it; \\ Tel: +39-011-090-6813, Fax: +39-011-090-6899.
}

Academic Editors: Luciano Feo and Raimondo Luciano

Received: 21 August 2015 / Accepted: 25 November 2015 / Published: 4 December 2015

\begin{abstract}
The present paper talks about the free vibration analysis of simply supported Single- and Double-Walled Carbon Nanotubes (SWCNTs and DWCNTs). Refined 2D Generalized Differential Quadrature (GDQ) shell methods and an exact 3D shell model are compared. A continuum approach (based on an elastic three-dimensional shell model) is used for natural frequency investigation of SWCNTs and DWCNTs. SWCNTs are defined as isotropic cylinders with an equivalent thickness and Young modulus. DWCNTs are defined as two concentric isotropic cylinders (with an equivalent thickness and Young modulus) which can be linked by means of the interlaminar continuity conditions or by means of van der Waals interactions. Layer wise approaches are mandatory for the analysis of van der Waals forces in DWCNTs. The effect of van der Waals interaction between the two cylinders is shown for different DWCNT lengths, diameters and vibration modes. The accuracy of beam models and classical 2D shell models in the free vibration analysis of SWCNTs and DWCNTs is also investigated.
\end{abstract}


Keywords: single-walled carbon nanotube; double-walled carbon nanotube; free vibrations; van der Waals interaction; three-dimensional shell model; exact solution; two-dimensional shell models; generalized differential quadrature method

\section{Introduction}

Research about Carbon NanoTubes (CNTs) has demonstrated their exceptional mechanical properties [1]. In view of these exceptional mechanical properties (the elastic modulus has been shown to be greater than $1 \mathrm{TPa}$ and the tensile strength exceeds that of steel by over one order of magnitude), CNTs are considered to be ideal reinforcements in composite structures [2]. CNTs are closed graphene sheets with a cylindrical shape, they were discovered in Japan by Iijma [3] in 1991. When a continuum elastic model is applied to CNT analysis, it is of central importance to accurately quantify the elastic properties of Single-Walled CNTs (SWCNTs) and Double-Walled CNTs (DWCNTs) [4].

The behavior of CNTs can be simulated by means of three different basic methods [5]: Molecular Dynamic (MD) simulations, atomistic-based modelling approaches and continuum approaches. In the MD approaches, the simulations are based on the definition of an appropriate potential energy function (e.g., Tersoff-Brenner or Lennard-Jones functions) [6-15]. In the atomistic-based modelling approaches, CNTs are investigated by means of an atomistic finite element model with beam elements and concentrated masses. The beams simulate the interatomic covalent forces and the masses (which are located at the ends of the beams) represent the carbon positions [16-21]. The continuum approaches are based on the assumption that carbon nanotubes (which have a discrete molecular structure) are continuum isotropic elastic cylinders which can be analyzed via beam or shell models. When a continuum elastic model is applied to CNT analysis, it is of central importance to accurately quantify the elastic properties of SWCNTs and DWCNTs [22,23].

The high computational cost of the MD simulations and the atomistic-based modelling approaches in the case of complex CNT networks does not allow fast analyses. Continuum approaches are preferred to MD and atomistic-based models because of their better computational cost. In order to apply a continuum model, it is necessary to correctly define effective CNT wall thickness, Young modulus and Poisson ratio even if a carbon nanotube has a discrete molecular structure. Extensive studies have been conducted to analyze this feature [24-27]. A final conclusion has not yet been reached, as demonstrated by the different thickness and Young modulus values shown in the papers analyzed in this contribution. The equivalent properties discussed in the papers proposed in the following review are not always the same for a given elastic stiffness.

Continuum approaches to analyze free vibrations of single-walled and multi-walled carbon nanotubes can use beam or shell models. The most important investigations about beam models for Single-Walled Carbon Nanotubes (SWCNTs) can be found in [28-41]. Further works about Double-Walled Carbon Nanotube (DWCNT) simulations via beam models can be found in [42-50]. Shell models are used for the analysis of SWCNTs and DWCNTs in [51-58] and [58-65], respectively.

The use of shell models for the vibration analysis of CNTs is usually more complicated than the use of beam models, but shell models allow the analysis of CNTs with small length/diameter ratios. 
For these structures, the use of 1D beam models gives significant errors because short CNTs are not one-dimensional structures. Refined 2D or 3D shell models are suitable for the correct vibration analysis of short CNTs, as demonstrated in [51,58], in particular when "the radius of curvature/thickness" ratio is small.

The present paper proposes an exact three-dimensional elastic shell model and several classical and refined two-dimensional elastic shell models solved by means of the Generalized Differential Quadrature (GDQ) method for free vibration analysis of simply supported SWCNTs and DWCNTs. The 3D exact shell model has been developed for the free vibration analysis of SWCNTs and DWCNTs in [51,59], respectively. The equilibrium equations in general orthogonal curvilinear coordinates (see [66-69]) are adapted to the case of a cylinder giving an infinite value for one of the two radii of curvature. The equilibrium equations in rectilinear orthogonal coordinates and in cylindrical coordinates were exactly solved by Messina [70] and Soldatos and Ye [71], respectively. Classical and refined two-dimensional GDQ methods for the free vibration analysis of shell structures have been developed in [72-81] where several benchmarks related to doubly-curved multilayered composite shells and higher-order theories have been proposed. The present GDQ method has been tested in [72-81] using 3D, 2D Finite Element Method (FEM) and semi-analytical solutions. In the present study, 2D GDQ models have been extended to the free vibration analysis of CNTs. Refined 2D GDQ models are based on the well-known unified formulation [82].

In order to apply the 3D or 2D shell continuum models, SWCNTs are defined as isotropic cylinders and DWCNTs are defined as two concentric isotropic cylinders (each cylinder in SWCNT or in DWCNT has an equivalent thickness and Young modulus). In DWCNTs, each cylinder can be linked by means of the interlaminar continuity conditions (first choice) or by means of an infinitesimal fictitious layer which represents the van der Waals interaction (second choice). The comparisons between these two choices show the effects of van der Waals interactions between the two concentric cylinders for different CNT lengths, diameters and vibration modes. Results show the van der Waals interaction effects in terms of frequency value. Shell and beam models, and 3D exact and 2D numerical shell models are compared for different SWCNT and DWCNT geometries.

\section{3D Exact Shell Model}

Free vibration analysis of multilayered spherical shells with constant radii of curvature $R_{\alpha}$ and $R_{\beta}$ has been proposed by Brischetto in [66-69] using three differential equations of equilibrium in general orthogonal curvilinear coordinates. The equations have been solved in exact form in analogy with the exponential matrix method proposed by Messina [70] and Soldatos and Ye [71] for orthogonal rectilinear coordinates and cylindrical coordinates, respectively. In the present paper, the equations in general orthogonal curvilinear coordinates are rewritten for the cylindrical case imposing an infinite value for the radius of curvature $R_{\beta}$ (see Figure 1). The general form proposed in [66-69] remains valid for both plate and constant radius shell geometries (spherical and cylindrical shells). 

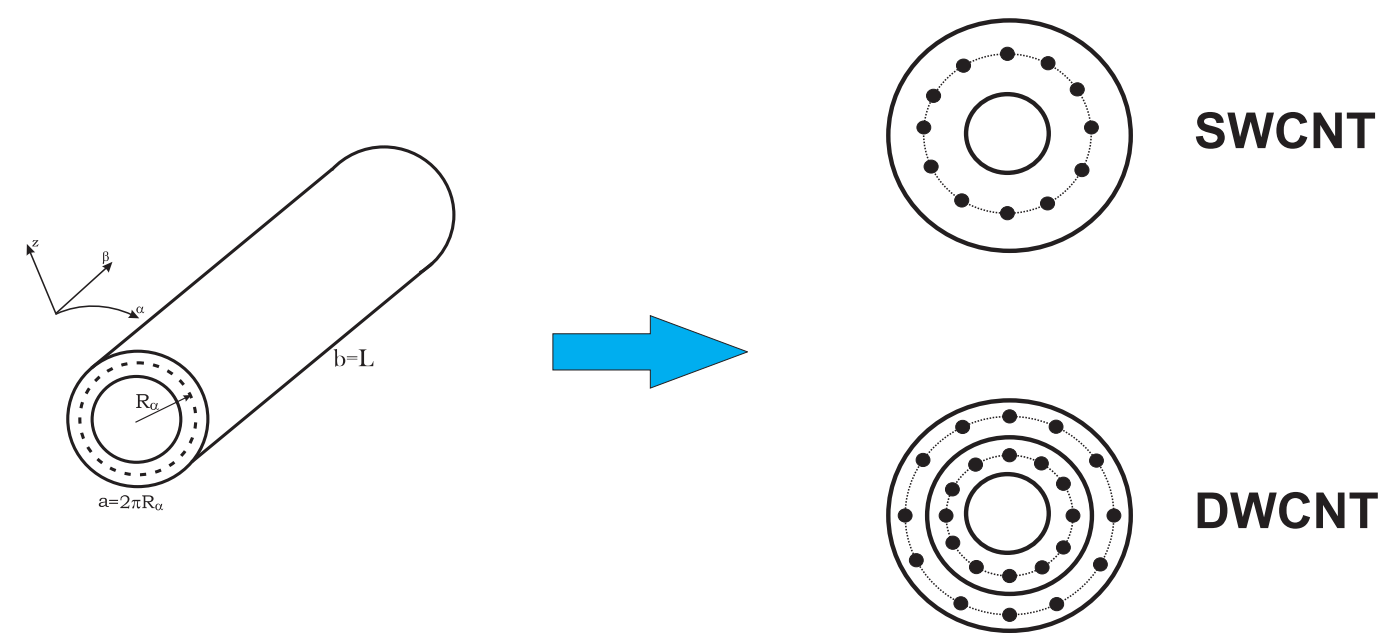

\section{DWCNT}

Figure 1. Reference system, notation and continuum approach for a SWCNT and a DWCNT.

The strain-displacement relations of the three-dimensional theory of elasticity in orthogonal curvilinear coordinates are written for the generic $k$ layer of the multilayered cylindrical shell of Figure 1 (the general form for spherical shells with constant radii of curvature $R_{\alpha}$ and $R_{\beta}$ has already been given in [66-69]):

$$
\begin{aligned}
\epsilon_{\alpha \alpha}^{k} & =\frac{1}{H_{\alpha}} u_{, \alpha}^{k}+\frac{w^{k}}{H_{\alpha} R_{\alpha}} \\
\epsilon_{\beta \beta}^{k} & =v_{, \beta}^{k} \\
\epsilon_{z z}^{k} & =w_{, z}^{k} \\
\gamma_{\beta z}^{k} & =w_{, \beta}^{k}+v_{, z}^{k} \\
\gamma_{\alpha z}^{k} & =\frac{1}{H_{\alpha}} w_{, \alpha}^{k}+u_{, z}^{k}-\frac{u^{k}}{H_{\alpha} R_{\alpha}} \\
\gamma_{\alpha \beta}^{k} & =\frac{1}{H_{\alpha}} v_{, \alpha}^{k}+u_{, \beta}^{k}
\end{aligned}
$$

The parametric coefficients for cylindrical shells are:

$$
H_{\alpha}=\left(1+\frac{z}{R_{\alpha}}\right), \quad H_{\beta}=1, \quad H_{z}=1
$$

The strain components are $\epsilon_{\alpha \alpha}, \epsilon_{\beta \beta}, \epsilon_{z z}, \gamma_{\beta z}, \gamma_{\alpha z}$ and $\gamma_{\alpha \beta}$ for each $k$ isotropic layer. The displacement components for each $k$ isotropic layer are $u, v$ and $w$ along orthogonal curvilinear coordinates $\alpha, \beta$ and $z$, respectively. Partial derivatives $\frac{\partial}{\partial \alpha}, \frac{\partial}{\partial \beta}$ and $\frac{\partial}{\partial z}$ are indicated with subscripts ${ }_{, \alpha}, \beta$ and ${ }_{, z}$, respectively. $H_{\alpha}$ depends on the $z$ coordinate. $H_{\beta}=1$ and $H_{z}=1$ because $\beta$ and $z$ are rectilinear coordinates. $R_{\alpha}$ is the principal radius of curvature along the $\alpha$ coordinate. $R_{\beta}$ is infinite for a cylinder (see Figure 1 ). 
Three-dimensional linear elastic constitutive equations in orthogonal curvilinear coordinates $(\alpha, \beta, z)$ (see Figure 1) are given for a generic $k$ isotropic layer. The stress components $\left(\sigma_{\alpha \alpha}, \sigma_{\beta \beta}, \sigma_{z z}, \sigma_{\beta z}, \sigma_{\alpha z}\right.$, $\left.\sigma_{\alpha \beta}\right)$ are linked with the strain components $\left(\epsilon_{\alpha \alpha}, \epsilon_{\beta \beta}, \epsilon_{z z}, \gamma_{\beta z}, \gamma_{\alpha z}, \gamma_{\alpha \beta}\right)$ for each $k$ isotropic layer as:

$$
\begin{aligned}
\sigma_{\alpha \alpha}^{k} & =C_{11}^{k} \epsilon_{\alpha \alpha}^{k}+C_{12}^{k} \epsilon_{\beta \beta}^{k}+C_{13}^{k} \epsilon_{z z}^{k} \\
\sigma_{\beta \beta}^{k} & =C_{12}^{k} \epsilon_{\alpha \alpha}^{k}+C_{22}^{k} \epsilon_{\beta \beta}^{k}+C_{23}^{k} \epsilon_{z z}^{k} \\
\sigma_{z z}^{k} & =C_{13}^{k} \epsilon_{\alpha \alpha}^{k}+C_{23}^{k} \epsilon_{\beta \beta}^{k}+C_{33}^{k} \epsilon_{z z}^{k} \\
\sigma_{\beta z}^{k} & =C_{44}^{k} \gamma_{\beta z}^{k} \\
\sigma_{\alpha z}^{k} & =C_{55}^{k} \gamma_{\alpha z}^{k} \\
\sigma_{\alpha \beta}^{k} & =C_{66}^{k} \gamma_{\alpha \beta}^{k}
\end{aligned}
$$

The most general form of differential equations of equilibrium for spherical shells with constant radii of curvature can be found in [66-69]. These equations rewritten for the case of free vibration analysis of cylindrical shells are:

$$
\begin{aligned}
& \sigma_{\alpha \alpha, \alpha}^{k}+H_{\alpha} \sigma_{\alpha \beta, \beta}^{k}+H_{\alpha} \sigma_{\alpha z, z}^{k}+\frac{2}{R_{\alpha}} \sigma_{\alpha z}^{k}=\rho^{k} H_{\alpha} \ddot{u}^{k} \\
& \sigma_{\alpha \beta, \alpha}^{k}+H_{\alpha} \sigma_{\beta \beta, \beta}^{k}+H_{\alpha} \sigma_{\beta z, z}^{k}+\frac{1}{R_{\alpha}} \sigma_{\beta z}^{k}=\rho^{k} H_{\alpha} \ddot{v}^{k} \\
& \sigma_{\alpha z, \alpha}^{k}+H_{\alpha} \sigma_{\beta z, \beta}^{k}+H_{\alpha} \sigma_{z z, z}^{k}-\frac{1}{R_{\alpha}} \sigma_{\alpha \alpha}^{k}+\frac{1}{R_{\alpha}} \sigma_{z z}^{k}=\rho^{k} H_{\alpha} \ddot{w}^{k}
\end{aligned}
$$

where $\rho^{k}$ is the mass density. $\ddot{u}^{k}, \ddot{v}^{k}$ and $\ddot{w}^{k}$ indicate the second temporal derivative of the three displacement components $u^{k}, v^{k}$ and $w^{k}$, respectively. Each quantity depends on the $k$ layer. $R_{\alpha}$ is referred to the mid-surface $\Omega_{0}$ of the whole multilayered shell. $H_{\alpha}$ continuously varies through the thickness of the multilayered shell and it depends on the $z$ thickness coordinate. Equations (14)-(16) have constant coefficients (even if a shell geometry is considered) when the shell is divided in $N_{L}$ mathematical layers where the parametric coefficient $H_{\alpha}$ can easily be calculated in the middle of each $k$ mathematical layer.

The closed form of Equations (14)-(16) is obtained for simply supported cylindrical shells. The three displacement components have the following harmonic form:

$$
\begin{aligned}
u^{k}(\alpha, \beta, z, t) & =U^{k}(z) e^{i \omega t} \cos (\bar{\alpha} \alpha) \sin (\bar{\beta} \beta) \\
v^{k}(\alpha, \beta, z, t) & =V^{k}(z) e^{i \omega t} \sin (\bar{\alpha} \alpha) \cos (\bar{\beta} \beta) \\
w^{k}(\alpha, \beta, z, t) & =W^{k}(z) e^{i \omega t} \sin (\bar{\alpha} \alpha) \sin (\bar{\beta} \beta)
\end{aligned}
$$

where $U^{k}(z), V^{k}(z)$ and $W^{k}(z)$ are the displacement amplitudes in $\alpha, \beta$ and $z$ directions, respectively. $i$ is the coefficient of the imaginary unit. $\omega=2 \pi f$ is the circular frequency where $f$ is the frequency value, $t$ is the time. In coefficients $\bar{\alpha}=\frac{p \pi}{a}$ and $\bar{\beta}=\frac{q \pi}{b}, p$ and $q$ are the half-wave numbers and $a$ and $b$ are the shell dimensions in $\alpha$ and $\beta$ directions, respectively (they are calculated in the reference mid-surface $\Omega_{0}$ ). 
Equations (1)-(6), (8)-(13) and (17)-(19) are substituted in Equations (14)-(16) to obtain a system of equations for each $k$ mathematical layer:

$$
\begin{aligned}
& A_{1}^{k} U^{k}+A_{2}^{k} V^{k}+A_{3}^{k} W^{k}+A_{4}^{k} U_{, z}^{k}+A_{5}^{k} W_{, z}^{k}+A_{6}^{k} U_{, z z}^{k}=0 \\
& A_{7}^{k} U^{k}+A_{8}^{k} V^{k}+A_{9}^{k} W^{k}+A_{10}^{k} V_{, z}^{k}+A_{11}^{k} W_{, z}^{k}+A_{12}^{k} V_{, z z}^{k}=0 \\
& A_{13}^{k} U^{k}+A_{14}^{k} V^{k}+A_{15}^{k} W^{k}+A_{16}^{k} U_{, z}^{k}+A_{17}^{k} V_{, z}^{k}+A_{18}^{k} W_{, z}^{k}+A_{19}^{k} W_{, z z}^{k}=0
\end{aligned}
$$

Coefficients $A_{s}^{k}$ are constant in each $k$ mathematical layer because parametric coefficient $H_{\alpha}$ is calculated in the middle of each $k$ layer.

Equations (20)-(22) are a system of three second order differential equations in $z$. This system can be reduced to a system of first order differential equations using the method already seen in [66-71]. In compact form, it can be written as:

$$
\boldsymbol{D}^{k} \frac{\partial \boldsymbol{U}^{k}}{\partial z}=\boldsymbol{A}^{k} \boldsymbol{U}^{k}
$$

where $\frac{\partial \boldsymbol{U}^{k}}{\partial z}=\boldsymbol{U}^{k^{\prime}}$ and $\boldsymbol{U}^{k}=\left[U^{k} V^{k} W^{k} U^{k^{\prime}} V^{k^{\prime}} W^{k^{\prime}}\right]$. Equation (23) can be rewritten as:

$$
\boldsymbol{U}^{k^{\prime}}=\boldsymbol{A}^{k^{*}} \boldsymbol{U}^{k}
$$

with $\boldsymbol{A}^{k^{*}}=\boldsymbol{D}^{k^{-1}} \boldsymbol{A}^{k}$. The solution of Equation (24) is obtained by means of the exponential matrix solution:

$$
\boldsymbol{U}^{k}\left(z^{k}\right)=\exp \left(\boldsymbol{A}^{k^{*}} z^{k}\right) \boldsymbol{U}^{k}(0) \quad \text { with } \quad z^{k} \epsilon\left[0, h^{k}\right]
$$

where $z^{k}$ is the thickness coordinate of each $k$ layer from 0 at the bottom to $h^{k}$ at the top.

If we consider $N_{L}$ layers, $N_{L}-1$ transfer matrices $\boldsymbol{T}^{k-1, k}$ must be calculated using for each interface the following conditions for interlaminar continuity of displacements and transverse shear/normal stresses:

$$
\begin{aligned}
& u_{b}^{k}=u_{t}^{k-1}, \quad v_{b}^{k}=v_{t}^{k-1}, \quad w_{b}^{k}=w_{t}^{k-1} \\
& \sigma_{z z b}^{k}=\sigma_{z z t}^{k-1}, \quad \sigma_{\alpha z b}^{k}=\sigma_{\alpha z t}^{k-1}, \quad \sigma_{\beta z b}^{k}=\sigma_{\beta z t}^{k-1}
\end{aligned}
$$

each displacement and transverse stress component at the top ( $\mathrm{t}$ ) of the $k-1$ layer is equal to each displacement and transverse stress component at the bottom (b) of the $k$ layer. Equations (26) and (27) in compact form are:

$$
\boldsymbol{U}_{b}^{k}=\boldsymbol{T}^{k-1, k} \boldsymbol{U}_{t}^{k-1}
$$

The calculated $\boldsymbol{T}^{k-1, k}$ matrices allow vector $\boldsymbol{U}$ at the bottom (b) of the $k$ layer with vector $\boldsymbol{U}$ at the top ( $\mathrm{t}$ ) of the $k-1$ layer to be linked. The structures are simply supported and free stressed at the top and at the bottom, this feature means:

$$
\begin{array}{lll}
\sigma_{z z}=\sigma_{\alpha z}=\sigma_{\beta z}=0 & \text { for } & z=0, h \\
w=v=0, \sigma_{\alpha \alpha}=0 & \text { for } & \alpha=0, a \\
w=u=0, \sigma_{\beta \beta}=0 & \text { for } & \beta=0, b
\end{array}
$$


The combination of Equations (24), (25), (28) and (29)-(31) leads to the following system (details can be found in [66-69]):

$$
\boldsymbol{E} \quad \boldsymbol{U}_{b}^{1}=\mathbf{0}
$$

Matrix $\boldsymbol{E}$ always has $(6 \times 6)$ dimension, independently from the number $N_{L}$ of mathematical layers, even if the method uses a layer-wise approach. The free vibration analysis means to find the non-trivial solution of $\boldsymbol{U}_{b}^{1}$ (displacement at the bottom of the layer 1) in Equation (32) imposing the determinant of matrix $\boldsymbol{E}$ equals zero:

$$
\operatorname{det}[\boldsymbol{E}]=0
$$

Equation (33) means to find the roots of an higher order polynomial in $\lambda=\omega^{2}$. For each pair of half-wave numbers $(p, q)$, a certain number of circular frequencies (from I to $\infty$ ) are obtained depending on the order $N$ chosen for the exponential matrix in Equation (25) and the number $N_{L}$ of mathematical layers.

\section{2D GDQ Shell Models}

The 2D GDQ models proposed in this manuscript consider an Equivalent Single Layer (ESL) and a Layer Wise (LW) approach, both developed in the framework of the well-known unified formulation [82]. The ESL models have the following displacement field:

$$
\mathbf{U}=\sum_{\tau=0}^{N_{c}+1} \mathbf{F}_{\tau} \mathbf{u}^{(\tau)}
$$

where $\mathbf{U}$ indicates the 3D displacement components and $\mathbf{u}$ stands for the vector of the $\tau$ th generalized displacements of the points on the middle surface of the shell [72]. $\mathbf{F}_{\tau(i j)}=\delta_{i j} F_{\tau}$ (for $i, j=1,2,3$ ) is the thickness function matrix and $\delta$ is the Kronecker delta function. A first order shear deformation theory, based on the Reissner-Mindlin model and called RM-GDQ, is obtained for $N_{c}=0$, and a higher-order model named ESL-GDQ is obtained with a fourth order expansion $\left(N_{c}=4\right)$. From the displacement field in Equation (34), the relation between generalized strains $\varepsilon^{(\tau)}$ and displacements $\mathbf{u}^{(\tau)}$ is:

$$
\boldsymbol{\varepsilon}^{(\tau)}=\mathbf{D}_{\Omega} \mathbf{u}^{(\tau)} \quad \text { for } \quad \tau=0,1,2, \ldots, N_{c}, N_{c}+1
$$

where $\mathbf{D}_{\Omega}$ is given in explicit form in [72]. The relationship between the $\tau^{t h}$ order stress resultants and the generalized strains is:

$$
\mathbf{S}^{(\tau)}=\sum_{s=0}^{N_{c}+1} \mathbf{A}^{(\tau s)} \boldsymbol{\varepsilon}^{(s)} \quad \text { for } \quad \tau=0,1,2, \ldots, N_{c}, N_{c}+1
$$

where

$$
\mathbf{A}^{(\tau s)}=\sum_{k=1}^{N_{L}} \int_{z_{k}}^{z_{k+1}}\left(\mathbf{Z}^{(\tau)}\right)^{T} \overline{\mathbf{C}}^{(k)} \mathbf{Z}^{(s)} H_{\alpha} H_{\beta} d z
$$

The elastic coefficients of the constitutive matrix are given in extended form in [72]. The governing equations of motion and their boundary conditions can be obtained via the Hamilton's Principle. In the 
present generalized approach, each order $\tau$ includes a set of three motion equations, that are a function of the internal actions and assume the following compact matrix form:

$$
\mathbf{D}_{\Omega}^{\star} \mathbf{S}^{(\tau)}=\sum_{s=0}^{N_{c}+1} \mathbf{M}^{(\tau s)} \ddot{\mathbf{u}}^{(s)} \quad \text { for } \quad \tau=0,1,2, \ldots, N_{c}, N_{c}+1,
$$

where $\mathbf{D}_{\Omega}^{\star}$ is the equilibrium operator and $\mathbf{M}^{(\tau s)}$ is the inertial matrix, they are given in explicit form in [72]. The mass matrix $\mathbf{M}_{(i j)}^{(\tau s)}=\delta_{i j} I_{0}^{(\tau s)}$ contains the inertial mass terms $I_{0}^{(\tau s)}$ for $i, j=1,2,3$ :

$$
I_{0}^{(\tau s)}=\sum_{k=1}^{N_{L}} \int_{z_{k}}^{z_{k+1}} \rho^{(k)} F_{\tau} F_{s} H_{\alpha} H_{\beta} d z \quad \text { for } \quad \tau, s=0,1,2, \ldots, N_{c}, N_{c}+1
$$

where $\rho^{(k)}$ represents the mass density of the material per unit of volume of the $k^{\text {th }}$ ply. Combining the kinematic Equation (35), constitutive Equation (36) and the motion Equation (38), the fundamental system of equations in terms of displacement parameters can be found:

$$
\sum_{s=0}^{N_{c}+1} \mathbf{L}^{(\tau s)} \mathbf{u}^{(s)}=\sum_{s=0}^{N_{c}+1} \mathbf{M}^{(\tau s)} \ddot{\mathbf{u}}^{(s)} \quad \text { for } \quad \tau=0,1,2, \ldots, N_{c}, N_{c}+1
$$

where $\mathbf{L}^{(\tau s)}=\mathbf{D}_{\Omega}^{\star} \mathbf{A}^{(\tau s)} \mathbf{D}_{\Omega}$ is the fundamental operator [72]. Boundary conditions must be introduced to solve the differential problem in Equation (40). The GDQ method works with any kind of boundary condition, in the present study only simply-supported edge boundary conditions (S) are taken into account in order to make comparisons with the 3D exact model:

$$
\begin{aligned}
& u_{\alpha}^{(\tau)}=0, N_{\beta}^{(\tau)}=0, u_{z}^{(\tau)}=0 \quad \text { for } \quad \tau=0,1,2, \ldots, N_{c}, N_{c}+1 \quad \text { at } \quad \beta=\beta^{0} \\
& \text { or } \beta=\beta^{1} \quad \alpha^{0} \leq \alpha \leq \alpha^{1}
\end{aligned}
$$

Along two edges, the simply-supported external boundary conditions are considered, whereas compatibility equations have been set on the other two edges in order to have a closed cylinder:

$$
\begin{array}{r}
u_{\alpha}^{(\tau)}(0, \beta, t)=u_{\alpha}^{(\tau)}(2 \pi, \beta, t) ; \quad u_{\beta}^{(\tau)}(0, \beta, t)=u_{\beta}^{(\tau)}(2 \pi, \beta, t) ; \quad u_{z}^{(\tau)}(0, \beta, t)=u_{z}^{(\tau)}(2 \pi, \beta, t) ; \\
N_{\alpha \beta}^{(\tau)}(0, \beta, t)=N_{\alpha \beta}^{(\tau)}(2 \pi, \beta, t) ; \quad N_{\alpha}^{(\tau)}(0, \beta, t)=N_{\alpha}^{(\tau)}(2 \pi, \beta, t) ; \quad T_{\alpha}^{(\tau)}(0, \beta, t)=T_{\alpha}^{(\tau)}(2 \pi, \beta, t) \\
\text { for } \tau=0,1,2, \ldots, N_{c}, N_{c}+1 \quad \text { at } \quad \beta^{0} \leq \beta \leq \beta^{1}
\end{array}
$$

The higher-order layer-wise model (LW-GDQ) has an approach similar to the ESL scheme. The displacement field takes the following form with $N_{c}=4$ [73]:

$$
\mathbf{U}^{(k)}=\sum_{\tau=0}^{N_{c}+1} \mathbf{F}_{\tau}^{(k)} \mathbf{u}^{(k \tau)} \quad \text { for } \quad k=1,2, \ldots, N_{L}
$$

From Equations (43) and (34), it is clear that each quantity is referred to each single layer $k$. In the present case, the thickness functions are a combination of Legendre polynomials recursively defined in [73]. The generalized displacements $u_{\alpha}^{(k 0)}, u_{\beta}^{(k 0)}, u_{z}^{(k 0)}$ for $\tau=0$ are the displacements at the bottom of the $k^{t h}$ layer $\left(z^{(k)}=-h_{k} / 2\right)$, whereas $u_{\alpha}^{\left(k\left(N_{c}+1\right)\right)}, u_{\beta}^{\left(k\left(N_{c}+1\right)\right)}, u_{z}^{\left(k\left(N_{c}+1\right)\right)}$ for $\tau=N_{c}+1$ are the 
displacements at the top of the $k^{\text {th }}$ layer $\left(z^{(k)}=+h_{k} / 2\right)$. Using the displacement field in Equation (43), the kinematic equations are:

$$
\varepsilon^{(k \tau)}=\mathbf{D}_{\Omega}^{(k)} \mathbf{u}^{(k \tau)} \quad \text { for } \quad \tau=0,1,2, \ldots, N_{c}, N_{c}+1, \quad k=1,2, \ldots, N_{L}
$$

A linear and elastic material constitutes the CNTs; thus, the present internal actions take the form:

$$
\mathbf{S}^{(k \tau)}=\sum_{s=0}^{N_{c}+1} \mathbf{A}^{(k \tau s)} \boldsymbol{\varepsilon}^{(k s)} \quad \text { for } \quad \tau=0,1,2, \ldots, N_{c}, N_{c}+1, \quad k=1,2, \ldots, N_{L}
$$

where

$$
\mathbf{A}^{(k \tau s)}=\sum_{k=1}^{N_{L}} \int_{-h_{k} / 2}^{+h_{k} / 2}\left(\mathbf{Z}^{(k \tau)}\right)^{T} \overline{\mathbf{C}}^{(k)} \mathbf{Z}^{(k s)} H_{\alpha}^{(k)} H_{\beta}^{(k)} d z^{(k)}
$$

The $\tau^{t h}$ order generalized internal action is indicated as $\mathbf{S}^{(k \tau)}$ and the elastic coefficients are $\mathbf{A}^{(k \tau s)}$, both computed as given in [73].

For the present layer-wise shell model, a set of three equilibrium equations for each order $\tau$ can be written:

$$
\mathbf{D}_{\Omega}^{\star(k)} \mathbf{S}^{(k \tau)}=\sum_{s=0}^{N_{c}+1} \mathbf{M}^{(k \tau s)} \ddot{\mathbf{u}}^{(k s)} \quad \text { for } \quad \tau=0,1,2, \ldots, N_{c}, N_{c}+1, \quad k=1,2, \ldots, N_{L}
$$

where the equilibrium operator $\mathbf{D}_{\Omega}^{\star(k)} \mathbf{S}^{(k \tau)}$ and the inertial matrix $\mathbf{M}^{(k \tau s)}$ have been explicitly shown in [73]. The inertial terms take place into the inertial matrix as $\mathbf{M}^{(k \tau s)}=\delta_{i j} I_{0}^{(k \tau s)}$ for $i, j=1,2,3$ and they are defined by the following expression:

$$
I_{0}^{(k \tau s)}=\int_{-h_{k} / 2}^{+h_{k} / 2} \rho^{(k)} F_{\tau}^{(k)} F_{s}^{(k)} H_{\alpha}^{(k)} H_{\beta}^{(k)} d z^{(k)} \quad \text { for } \quad \tau, s=0,1,2, \ldots, N_{c}, N_{c}+1, \quad k=1,2, \ldots, N_{L}
$$

where $\rho^{(k)}$ is the mass density of the material per unit of volume of the $k^{t h}$ lamina. Finally, the fundamental equations in terms of generalized displacements are:

$$
\sum_{s=0}^{N_{c}+1} \mathbf{L}^{(k \tau s)} \mathbf{u}^{(k s)}=\sum_{s=0}^{N_{c}+1} \mathbf{M}^{(k \tau s)} \ddot{\mathbf{u}}^{(k s)} \quad \text { for } \quad \tau=0,1,2, \ldots, N_{c}, N_{c}+1, \quad k=1,2, \ldots, N_{L}
$$

where $\mathbf{L}^{(k \tau s)}=\mathbf{D}_{\Omega}^{\star(k)} \mathbf{A}^{(k \tau s)} \mathbf{D}_{\Omega}^{(k)}$ is the fundamental operator [73]. Since the approach is based on a layer-by-layer structure, the compatibility conditions between the layers must be defined. In detail, the top displacements of the $k^{\text {th }}$ ply at each interface must be equal to the bottom displacements of the $(k+1)^{t h}$ layer, as:

$$
\left[\begin{array}{l}
u_{\alpha}^{(k \text { top })} \\
u_{\beta}^{(k \text { top })} \\
u_{z}^{(k \text { top })}
\end{array}\right]=\left[\begin{array}{l}
u_{\alpha}^{((k+1) \text { bottom })} \\
u_{\beta}^{((k+1) \text { bottom })} \\
u_{z}^{((k+1) \text { bottom })}
\end{array}\right] \rightarrow\left[\begin{array}{l}
u_{\alpha}^{\left(k\left(N_{c}+1\right)\right)} \\
u_{\beta}^{\left(k\left(N_{c}+1\right)\right)} \\
u_{z}^{\left(k\left(N_{c}+1\right)\right)}
\end{array}\right]=\left[\begin{array}{l}
u_{\alpha}^{((k+1) 0)} \\
u_{\beta}^{((k+1) 0)} \\
u_{z}^{((k+1) 0)}
\end{array}\right] \quad \text { for } \begin{aligned}
& \tau=0,1,2, \ldots, N_{c}, N_{c}+1 \\
& k=1,2, \ldots, N_{L}-1
\end{aligned}
$$

Finally, boundary conditions must be included to solve the equations. In the present study, only simply-supported boundary conditions have been applied because of the comparison with the 3D 
exact solution (even though GDQ method based on the LW approach can work with any kind of boundary condition):

$$
u_{\alpha}^{(k \tau)}=0, N_{\beta}^{(k \tau)}=0, u_{z}^{(k \tau)}=0 \quad \text { for } \quad \begin{aligned}
& \tau=0,1,2, \ldots, N_{c}, N_{c}+1 \\
& k=1,2, \ldots, N_{L}
\end{aligned} \quad \text { at } \quad \begin{aligned}
& \beta=\beta^{0} \quad \text { or } \beta=\beta^{1} \\
& \alpha^{0} \leq \alpha \leq \alpha^{1}
\end{aligned}
$$

Since a closed cylinder has been investigated, compatibility conditions must be considered, they are given in the following form:

$$
\begin{array}{r}
u_{\alpha}^{(k \tau)}(0, \beta, t)=u_{\alpha}^{(k \tau)}(2 \pi, \beta, t) ; \quad u_{\beta}^{(k \tau)}(0, \beta, t)=u_{\beta}^{(k \tau)}(2 \pi, \beta, t) ; \quad u_{z}^{(\tau)}(0, \beta, t)=u_{z}^{(\tau)}(2 \pi, \beta, t) ; \\
N_{\alpha \beta}^{(k \tau)}(0, \beta, t)=N_{\alpha \beta}^{(k \tau)}(2 \pi, \beta, t) ; \quad N_{\alpha}^{(k \tau)}(0, \beta, t)=N_{\alpha}^{(k \tau)}(2 \pi, \beta, t) ; \quad T_{\alpha}^{(k \tau)}(0, \beta, t)=T_{\alpha}^{(k \tau)}(2 \pi, \beta, t) \\
\text { for } \tau=0,1,2, \ldots, N_{c}, N_{c}+1 \quad \text { at } \quad \beta^{0} \leq \beta \leq \beta^{1}
\end{array}
$$

\section{Results and Discussion}

This section proposes results for free vibration analysis of simply supported SWCNTs and DWCNTs. The exact 3D solution described in Section 2 will be compared with two beam models proposed in the literature by Simsek [39] and Aydogdu [20], and with the 2D GDQ solutions described in Section 3 (in particular a Reissner-Mindlin model (RM-GDQ), a refined Equivalent Single Layer model (ESL-GDQ) and a refined Layer Wise model (LW-GDQ)). A preliminary assessment is given to validate the 3D exact and the 2D GDQ models; in this case, several SWCNTs are analyzed. The length $L$ of the investigated SWCNTs is changed to understand the approximation of the beam models. These preliminary assessments have been used to understand the order $N$ for the exponential matrix and the number of mathematical layers $N_{L}$ to use in the 3D exact model, and the number of grid points to use in 2D GDQ models. In the benchmarks for SWCNTs and DWCNTs, 2D GDQ models are compared with the 3D exact model for circumferential half-wave number $p=2$ and longitudinal half-wave numbers $q=1,2,3$ (the first mode (I) for each pair $(p, q)$ is always indicated in the tables). The comparison between the 3D exact model and the 2D GDQ models is possible by means of an appropriate vibration mode analysis which allows to select the appropriate frequencies and half-wave numbers. The effects of the van der Waals forces have been evaluated by means of the 3D exact model which is based on a layer wise approach.

\subsection{Preliminary Assessment}

This assessment has been proposed by Simsek [39] and Aydogdu [20] where an Euler-Bernoulli Beam Model (EBM) and a Timoshenko Beam Model (TBM) have been used. The SWCNT is simply supported, the equivalent elastic cylinder has properties as indicated in Simsek [39]. The equivalent Young modulus is $E=1 \mathrm{TPa}$ with Poisson ratio $\nu=0.3$, the effective thickness considered for this Young modulus value is $h=0.35 \mathrm{~nm}$. The mass density is $\rho=2300 \mathrm{~kg} / \mathrm{m}^{3}$. The external diameter of the cylinder is $d_{e}=1 \mathrm{~nm}$, this value means a "radius of curvature/thickness" ratio $R_{\alpha} / h=0.929$ (very thick shell) which requests the use of beam models or 3D/refined 2D shell models. Some difficulties may arise when classical 2D shell models (e.g., RM-GDQ) are used for the analysis of such cylinders. The use of very refined 2D shell models (see [58]) or 3D exact shell models (see [51,59]) overcomes this problem. The radius of curvature in $\alpha$ direction, referred to the mid-surface, is $R_{\alpha}=d_{e} / 2-h / 2=0.325 \mathrm{~nm}$. The 
dimension in $\alpha$ direction is $a=2 \pi R_{\alpha}$, and the $b$ dimension is $L=5 \mathrm{~nm}, 10 \mathrm{~nm}, 20 \mathrm{~nm}, 50 \mathrm{~nm}$ and 100 $\mathrm{nm}$ for ratios $L / d_{e}=5,10,20,50$ and 100, respectively. Table 1 gives the non-dimensional circular frequency $\bar{\omega}=\omega L^{2} \sqrt{\frac{\rho A}{E I}}$ (where $A=\pi\left(r_{e}^{2}-r_{i}^{2}\right)$ is the area of the ring and $I=\frac{\pi}{64}\left(d_{e}^{4}-d_{i}^{4}\right)$ is the moment of inertia of the ring) for short and long simply supported cylinders with different $L / d_{e}$ ratios. The three non-dimensional circular frequencies are obtained with an imposed half-wave number $p=2$ in $\alpha$ direction and half-wave numbers $q$ in $\beta$ direction equal to 1,2 and 3 (the first mode (I) is considered for each pair $(p, q)$ ). Beam models correctly work for long and moderately long cylinders. However, shell models give correct results for both long and short cylinders. The Euler-Bernoulli Beam Model (EBM) was proposed in Simsek [39] and Aydogdu [20] for ratios $L / d_{e}=10,20,50$. The same cases were also investigated in [20] by means of the Timoshenko Beam Model (TBM). TBM gives more accurate results than EBM because it includes the effects of transverse shear deformation and rotary inertia. However, TBM shows some problems for second and third frequency in the case of short SWCNTs $\left(L / d_{e}=10\right)$. The 3D shell model gives satisfactory results for both long and short SWCNTs, and it also allows for the vibration analysis of cylinders with small "radius of curvature/thickness" ratios. For these small ratios, classical 2D shell models could exhibit some difficulties. Table 1 shows that the TBM gives similar results to the 3D shell model, while the EBM produces larger differences. The TBM has some difficulties for short SWCNTs. Additional results for very short and very long SWCNTs via the 3D shell model and 2D GDQ models are recorded in Table 1 (they were not obtained in [20,39] via beam models). They show a complete overview of the SWCNT behavior, and they can be used as a benchmark for the validation of future 1D beam and 2D shell models. 2D GDQ models propose both classical (Reissner-Mindlin approach, RM-GDQ) and refined (higher order Equivalent Single Layer approach, ESL-GDQ) models. ESL-GDQ model always gives the 3D exact solution for each $L / d_{e}$ ratio (length of SWCNT) and vibration mode. RM-GDQ exhibits some difficulties because the cylinder is very thick ( $R_{\alpha} / h=0.929$ ). The layer wise model (LW-GDQ) is not used in this assessment because only one layer is embedded in the SWCNT. Scientists involved in beam and shell model analyses of SWCNTs can try to complete this table. After this assessment, the 3D shell model and the 2D GDQ models can be considered as validated and it can be used with confidence for future analyses. The 3D shell model used $N_{L}=228$ mathematical layers for the approximation of the radius of curvature and $N=3$ order for the approximation of the exponential matrix. 2D GDQ models used a $41 \times 15$ Chebyshev-Gauss-Lobatto grid. All these values are also used in the benchmark analysis of Section 4.2. 
Table 1. Preliminary assessment, simply supported single-walled carbon nanotubes (SWCNT) with $R_{\alpha} / h=0.929$. Comparison between the 3D exact and 2D GDQ shell models and beam models (Euler-Bernoulli Beam Model (EBM) and Timoshenko Beam Model (TBM) proposed in [20,39]). Non-dimensional circular frequencies $\bar{\omega}$ for different $L / d_{e}$ ratios. $p$ and $q$ are the imposed half-wave numbers.

\begin{tabular}{|c|c|c|c|c|c|}
\hline mode $(p, q)$ & 3D Exact & RM-GDQ & ESL-GDQ & EBM [20,39] & TBM[20] \\
\hline \multicolumn{6}{|c|}{$L / d_{e}=5$} \\
\hline $\mathrm{I}(2,1)$ & 9.3481 & 9.3672 & 9.3480 & - & - \\
\hline $\mathrm{I}(2,2)$ & 32.917 & 32.941 & 32.907 & - & - \\
\hline I $(2,3)$ & 63.917 & 63.683 & 63.685 & - & - \\
\hline \multicolumn{6}{|c|}{$L / d_{e}=10$} \\
\hline $\mathrm{I}(2,1)$ & 9.7295 & 9.7525 & 9.7290 & 9.8696 & 9.7443 \\
\hline $\mathrm{I}(2,2)$ & 37.392 & 37.469 & 37.392 & 39.478 & 36.841 \\
\hline $\mathrm{I}(2,3)$ & 79.361 & 79.483 & 79.358 & 88.826 & 57.450 \\
\hline \multicolumn{6}{|c|}{$L / d_{e}=20$} \\
\hline I $(2,1)$ & 9.8356 & 9.8584 & 9.8337 & 9.8696 & 9.8381 \\
\hline $\mathrm{I}(2,2)$ & 38.918 & 39.010 & 38.916 & 39.478 & 38.964 \\
\hline I $(2,3)$ & 86.072 & 86.264 & 86.070 & 88.826 & 85.748 \\
\hline \multicolumn{6}{|c|}{$L / d_{e}=50$} \\
\hline $\mathrm{I}(2,1)$ & 9.8638 & 9.8888 & 9.8638 & 9.8696 & 9.8645 \\
\hline $\mathrm{I}(2,2)$ & 39.392 & 39.485 & 39.386 & 39.478 & 39.398 \\
\hline $\mathrm{I}(2,3)$ & 88.375 & 88.583 & 88.363 & 88.826 & 88.415 \\
\hline \multicolumn{6}{|c|}{$L / d_{e}=100$} \\
\hline $\mathrm{I}(2,1)$ & 9.8487 & 9.8932 & 9.8682 & - & - \\
\hline $\mathrm{I}(2,2)$ & 39.488 & 39.555 & 39.455 & - & - \\
\hline $\mathrm{I}(2,3)$ & 88.752 & 88.934 & 88.710 & - & - \\
\hline
\end{tabular}

\subsection{Benchmarks}

The first benchmark considers a simply supported SWCNT with properties proposed in [6] and in Table 2 and Figure 2. The SWCNTs can have different geometries depending on the chiral vector $\overrightarrow{C_{h}}=n \overrightarrow{a_{1}}+m \overrightarrow{a_{2}}$ which also gives different values of the radius of curvature (see Figure 2 and Table 2 for further details about armachair, zigzag and general chirality CNTs). Chen and Cao [6] proposed different continuum approaches for CNT analysis, they gave a completely different value for the thickness wall $h$ with respect to those usually proposed in the literature. This value was $h=0.08 \mathrm{~nm}$ that means an effective Young modulus $E=6.85 \mathrm{TPa}$ and Poisson ratio $\nu=0.19$. The mass density 
is $\rho=9517 \mathrm{~kg} / \mathrm{m}^{3}$. The continuum models proposed in [6] used these mechanical properties for the CNT analysis. The comparison of these results with those obtained via the Molecular Dynamic (MD) analysis has always shown a difference which is less than $5 \%$. For this reason, these properties have been used for the SWCNT benchmark proposed in this section by means of the 3D exact model and the 2D GDQ models. Table 2 shows the radius of curvature $r$ and the number of atoms for several SWCNT configurations (armachair with $n=m$, zigzag with $(n, 0)$ and general chirality with $n \neq m$ ). Several "length/radius" ratios $(L / r)$ are proposed for each SWCNT, $r=R_{\alpha}$ is considered as the radius of curvature in the $\alpha$ direction with respect to the mid-surface. Tables 3-5 show the frequencies in $\mathrm{GHz}$ for each SWCNT described in Table 2 and Figure 2 with "length/radius" ratios $L / r=20,25,30,35$. The $R_{\alpha} / h$ ratio is bigger than the ratio proposed in the preliminary assessment (moderately thick shell), for this reason the classical 2D GDQ model works as well as the refined 2D GDQ model and the 3D exact model. Only SWCNTs are considered in this first benchmark, therefore the use of 2D LW models is not necessary. The 3D exact model is compared with the 2D GDQ model based on the Reissner-Mindilin approach (RM-GDQ) and the 2D GDQ model based on a fourth order Equivalent Single Layer model (ESL-GDQ). 2D GDQ models give a number of frequencies equal to the number of degrees of freedom of the employed model. In Tables 3-5, the comparisons are made with the frequencies given by the 3D exact model when the circumferential half-wave number is $p=2$ and the longitudinal half-wave number $q$ is equal to 1, 2 and 3 (always the first mode (I) for each pair $(p, q)$ ). This comparison is possible via the visualization of the vibration modes of the 2D GDQ models, which allows one to understand the values of $p$ and $q$. In Tables 3-5, 3D exact frequencies are always very similar to frequencies obtained by means of 2D GDQ models (both classical RM-GDQ and refined ESL-GDQ ones) for each $L / r$ ratio investigated, a couple of half-wave numbers $(p, q)$ and SWCNT structure (armachair, zigzag and general chirality). This feature is due to the fact that this SWCNT is a one-layered moderately thick isotropic cylinder. For a smaller "radius of curvature/thickness" ratio (thick shells), the use of 3D or refined 2D shell models will be mandatory.

Table 2. Geometry and properties (radius of curvature and number of atoms) for some of the SWCNTs used in the present paper and in Chen and Cao work [6].

\begin{tabular}{llll}
\hline SWCNT (n,m) & $\mathbf{( 5 , 5 )}$ & $\mathbf{( 1 0 , 0 )}$ & $\mathbf{( 8 , 4 )}$ \\
\hline r(nm) & 0.338 & 0.390 & 0.413 \\
n. atoms & 1040 & 1200 & 1200 \\
\hline
\end{tabular}



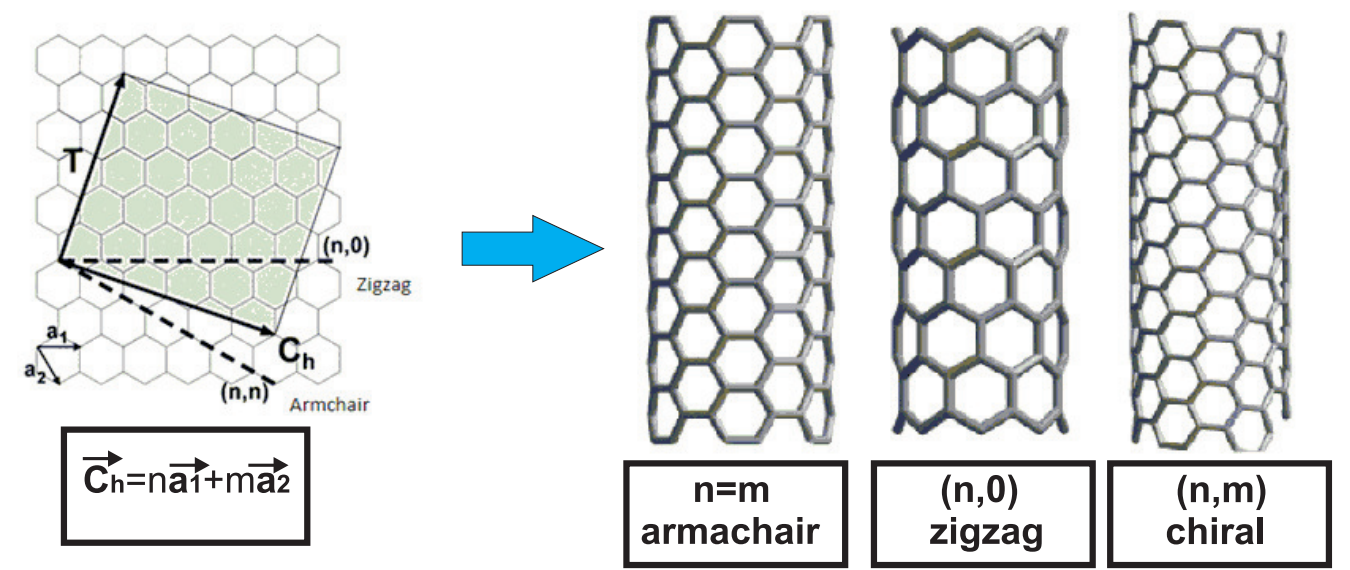

Figure 2. Notation of the chiral vector for armachair, zigzag and general chirality single-walled carbon nanotubes.

Table 3. First benchmark, simply supported armachair SWCNT with $R_{\alpha} / h=4.225$. Frequencies in GHz for 2D generalized differential quadrature (GDQ) and 3D exact shell models.

\begin{tabular}{cccc}
\hline \multicolumn{5}{c}{ Armachair (5,5) } \\
\hline mode (p,q) & 3D Exact & RM-GDQ & ESL-GDQ \\
\hline \multicolumn{4}{c}{$L / r=20$} \\
\hline I $(2,1)$ & 215.0 & 215.0 & 215.0 \\
I $(2,2)$ & 791.7 & 791.8 & 791.7 \\
I $(2,3)$ & 1595 & 1595 & 1595 \\
\hline \multicolumn{5}{c}{$L / r=25$} \\
\hline I $(2,1)$ & 139.1 & 139.1 & 139.2 \\
I $(2,2)$ & 526.2 & 526.2 & 526.2 \\
I $(2,3)$ & 1093 & 1093 & 1093 \\
\hline \multicolumn{5}{c}{$L / r=30$} \\
\hline I $(2,1)$ & 97.23 & 97.24 & 97.23 \\
I $(2,2)$ & 373.5 & 373.5 & 373.5 \\
I $(2,3)$ & 791.7 & 791.8 & 791.7 \\
\hline \multicolumn{5}{c}{$L / r=35$} \\
\hline I $(2,1)$ & 71.70 & 71.71 & 71.70 \\
I $(2,2)$ & 278.2 & 278.3 & 278.2 \\
I $(2,3)$ & 597.9 & 597.9 & 597.9 \\
\hline
\end{tabular}


Table 4. First benchmark, simply supported zigzag SWCNT with $R_{\alpha} / h=4.875$. Frequencies in GHz for 2D GDQ and 3D exact shell models.

\begin{tabular}{cccc}
\hline \multicolumn{4}{c}{ Zigzag (10,0) } \\
\hline Mode (p,q) & 3D Exact & RM-GDQ & ESL-GDQ \\
\hline \multicolumn{4}{c}{$L / r=20$} \\
\hline I $(2,1)$ & 186.0 & 186.0 & 186.0 \\
I $(2,2)$ & 684.9 & 684.9 & 684.9 \\
I $(2,3)$ & 1380 & 1380 & 1380 \\
\hline \multicolumn{5}{c}{$L / r=25$} \\
\hline I $(2,1)$ & 120.4 & 120.4 & 120.4 \\
I $(2,2)$ & 455.2 & 455.2 & 455.2 \\
I $(2,3)$ & 945.7 & 945.7 & 945.7 \\
\hline \multicolumn{5}{c}{$L / r=30$} \\
\hline I $(2,1)$ & 84.12 & 84.12 & 84.12 \\
I $(2,2)$ & 323.1 & 323.2 & 323.1 \\
I $(2,3)$ & 684.9 & 685.0 & 684.9 \\
\hline \multicolumn{5}{c}{$L / r=35$} \\
\hline I $(2,1)$ & 62.04 & 62.04 \\
I $(2,2)$ & 240.7 & 240.7 & 62.04 \\
I $(2,3)$ & 517.2 & 517.2 \\
\hline
\end{tabular}

Table 5. First benchmark, simply supported SWCNT with general chirality and $R_{\alpha} / h=5.1625$. Frequencies in GHz for 2D GDQ and 3D exact shell models.

\begin{tabular}{|c|c|c|c|}
\hline \multicolumn{4}{|c|}{ General Chirality $(\mathbf{8 , 4 )}$} \\
\hline $\operatorname{Mode}(\mathbf{p}, \mathbf{q})$ & 3D Exact & RM-GDQ & ESL-GDQ \\
\hline \multicolumn{4}{|c|}{$L / r=20$} \\
\hline $\mathrm{I}(2,1)$ & 175.5 & 175.6 & 175.5 \\
\hline $\mathrm{I}(2,2)$ & 646.4 & 646.4 & 646.4 \\
\hline I $(2,3)$ & 1302 & 1302 & 1302 \\
\hline \multicolumn{4}{|c|}{$L / r=25$} \\
\hline $\mathrm{I}(2,1)$ & 113.6 & 113.6 & 113.6 \\
\hline I $(2,2)$ & 429.6 & 429.6 & 429.6 \\
\hline I $(2,3)$ & 892.4 & 892.5 & 892.4 \\
\hline \multicolumn{4}{|c|}{$L / r=30$} \\
\hline I $(2,1)$ & 79.39 & 79.39 & 79.39 \\
\hline $\mathrm{I}(2,2)$ & 305.0 & 305.0 & 305.0 \\
\hline $\mathrm{I}(2,3)$ & 646.4 & 646.4 & 646.4 \\
\hline \multicolumn{4}{|c|}{$L / r=35$} \\
\hline $\mathrm{I}(2,1)$ & 58.55 & 58.55 & 58.55 \\
\hline $\mathrm{I}(2,2)$ & 227.2 & 227.2 & 227.2 \\
\hline I $(2,3)$ & 488.1 & 488.1 & 488.1 \\
\hline
\end{tabular}

The second benchmark considers four different DWCNTs. The DWCNT has two layers with the same equivalent thickness $h_{i}=h_{e}=0.35 \mathrm{~nm}$ (see Figures 1, 3 and 4). The equivalent elastic 
properties are Young modulus $E=1 \mathrm{TPa}$ and Poisson ratio $\nu=0.25$. The mass density is $\rho=2300 \mathrm{~kg} / \mathrm{m}^{3}$. Four different DWCNT geometries are investigated (see Figures 1 and 4 for reference values). The first nanotube has a reference diameter for the inner cylinder $d_{i}=0.7 \mathrm{~nm}$ and a reference diameter for the external cylinder $d_{e}=1.4 \mathrm{~nm}$. The mean radius of curvature for the DWCNT is $R_{\alpha}=d_{i} / 2+h_{i} / 2=0.525 \mathrm{~nm}$; this value means dimension $a=2 \pi R_{\alpha}=3.298672286 \mathrm{~nm}$. The second nanotube has a reference diameter for the inner cylinder $d_{i}=2.95 \mathrm{~nm}$ and a reference diameter for the external cylinder $d_{e}=3.65 \mathrm{~nm}$. The mean radius of curvature for the DWCNT is $R_{\alpha}=d_{i} / 2+h_{i} / 2=1.65 \mathrm{~nm}$; this value means dimension $a=2 \pi R_{\alpha}=10.367256 \mathrm{~nm}$. The third nanotube has a reference diameter for the inner cylinder $d_{i}=4.80 \mathrm{~nm}$ and a reference diameter for the external cylinder $d_{e}=5.50 \mathrm{~nm}$. The mean radius of curvature for the DWCNT is $R_{\alpha}=d_{i} / 2+h_{i} / 2=2.575 \mathrm{~nm}$; this value means dimension $a=2 \pi R_{\alpha}=16.1792022 \mathrm{~nm}$. The fourth nanotube has a reference diameter for the inner cylinder $d_{i}=7.0 \mathrm{~nm}$ and a reference diameter for the external cylinder $d_{e}=7.70 \mathrm{~nm}$. The mean radius of curvature for the DWCNT is $R_{\alpha}=d_{i} / 2+h_{i} / 2=3.675 \mathrm{~nm}$; this value means dimension $a=2 \pi R_{\alpha}=23.090706 \mathrm{~nm}$. The four DWCNTs have an infinity radius of curvature in $\beta$ direction. The lengths $L=b$ considered in the $\beta$ direction are obtained from $L / d_{e}=5,10,15,30$ and 50. The DWCNT is analyzed in this paper by means of an equivalent continuum model where the two cylinders have thickness values $h_{i}$ and $h_{e}$ (see Figures 3 and 4). The first 3D exact model considers $N_{L}=228$ mathematical layers to correctly approximate the curvature of the shell. The first cylinder (with thickness $h_{i}$ ) is divided into 114 mathematical layers (from $k=1$ to $k=114$ ) and the second cylinder (with thickness $h_{e}$ ) is also divided into 114 mathematical layers (from $k=115$ to $k=228$ ). Mathematical layers are linked by means of the interlaminar continuity conditions given in Equations (26) and (27). Such conditions are also used to link layer $k=114$ to layer $k=115$, in this way the two cylinders are linked by means of interlaminar continuity conditions. This model is indicated as the 3D model in the proposed results. ESL-GDQ and LW-GDQ models consider the DWCNT as including two layers with thickness $h_{i}=h_{e}=0.35 \mathrm{~nm}$, ESL-GDQ is based on an Equivalent Single Layer approach and LW-GDQ is based on a Layer Wise approach that links the two cylinders by means of the interlaminar continuity conditions. The second 3D exact model proposed in this paper is called the $3 \mathrm{D}_{v d W}$ model because it allows van der Waals interactions to be included in the 3D continuum shell model described in Section 2. The two cylinders have thickness values $h_{i}$ and $h_{e}$. The first cylinder is divided into 114 fictitious layers (from $k=1$ to $k=114$ ) and the second cylinder is also divided into 114 fictitious layers (from $k=116$ to $k=229$ ). An infinitesimal layer $(k=115)$ is introduced between the two cylinders to simulate the van der Waals interaction (see Figure 3). This infinitesimal fictitious layer has a negligible thickness $\left(h_{115}=\left(h_{i}+h_{e}\right) / 1000\right)$ and opportune elastic properties which represent the van der Waals interaction. Layers $k=114$ and $k=115$, and layers $k=115$ and $k=116$ are linked by means of the interlaminar continuity conditions given in Equations (26) and (27). The fictitious layer $k=115$ has mass density $\rho=1.225 \mathrm{~kg} / \mathrm{m}^{3}$ (air density). The Poisson ratio is the same used for the other layers. The van der Waals interaction coefficient $c$, estimated at the initial interlayer spacing can be given as:

$$
c=\frac{320 \mathrm{erg} / \mathrm{cm}^{2}}{0.16 d^{2}}
$$


see de Borbón and Ambrosini [48] for further details. $d=0.142 \mathrm{~nm}$ is the length of $\mathrm{C}-\mathrm{C}$ bond and $1 \mathrm{erg}=10^{-7}$ Joule. This coefficient has value $c=9.918667 \times 10^{19} \mathrm{~N} / \mathrm{m}^{3}$. Transverse displacements for CNT analysis have values in the order of $10^{-12} \mathrm{~m}$. Therefore, the equivalent Young modulus for the infinitesimal fictitious layer that simulates van der Waals interactions could be $E=9.918667 \times 10^{7} \mathrm{~Pa}$. An alternative method could be the inclusion of van der Waals forces directly in Equations (14)-(16) of the $3 \mathrm{D}$ exact model. The method of the fictitious layer has been preferred in the $3 \mathrm{D}$ exact model because the direct inclusion of van der Waals forces gave numerical problems in the solution of the 3D exact equations. The method including the van der Waals forces and their coefficients $\mathrm{c}$ has been used by the first author in [58] in the case of refined 2D models. The method using the fictitious layer, that represents the van der Waals forces, has been validated and tested in [59] by the first author for the 3D exact model. Tables 6-9 show results for the four different DWCNT geometries described in this section. Comparisons are made between the 3D exact model and the refined 2D GDQ models for several length-to-diameter ratio (from very short DWCNTs to very long DWCNTs). 2D GDQ models are used to obtain the $3 \mathrm{D}$ exact frequencies (only the first mode I) when $p=2$ and $q=1,2,3$. 2D GDQ models give several frequencies. In order to understand which are the frequencies to compare, a vibration mode analysis must be performed as shown in the examples proposed in Figure 5. This analysis allows to understand the values of half-wave numbers $(p, q)$. In Tables 6-9, 3D exact frequencies are always very similar to results obtained via refined 2D GDQ models. RM-GDQ model is not given because it shows big errors for cylinders with small $R_{\alpha} / h$ values (in particular Tables 6 and 7) and it is coincident with the refined 2D GDQ models with big $R_{\alpha} / h$ values (in particular Tables 8 and 9). Both refined 2D GDQ models (the Equivalent Single Layer one and the Layer Wise one) give always the same results even if a double-walled CNT is considered. This feature is due to the fact that the two cylinders are isotropic and made of the same material, and, for these reasons, ESL and LW approaches are coincident. Refined 2D GDQ models are always very accurate for each DWCNT geometry, "length/diameter" ratio, "radius of curvature/thickness" ratio and vibration mode. In Tables 6-9, a 3. $\mathrm{D}_{v d W}$ exact model is also considered. It allows to evaluate the van der Waals interaction effects by means of the frequency comparison between the 3D exact model (which considers the interlaminar continuity conditions between the two cylinders) and the $3 \mathrm{D}_{v d W}$ exact model (which considers a fictitious layer between the two cylinders that represents the van der Waals forces). From Tables 6-9, it is clear how the van der Waals effects are usually very small (often negligible). These effects are quite zero for long DWCNTs and/or low frequencies ( $p=2$ and $q=1$ ), while they are more important for short cylinders and/or high frequencies ( $p=2$ and $q=2$ or 3 ). van der Waals interaction effects are bigger in Tables 6 and 7 because they consider cylinders with a small $R_{\alpha} / h$ ratio. For cylinders in Tables 8 and 9 (bigger $R_{\alpha} / h$ ratios), van der Waals effects are always negligible for each lenght $\left(L / d_{e}\right.$ ratio) and vibration mode. 


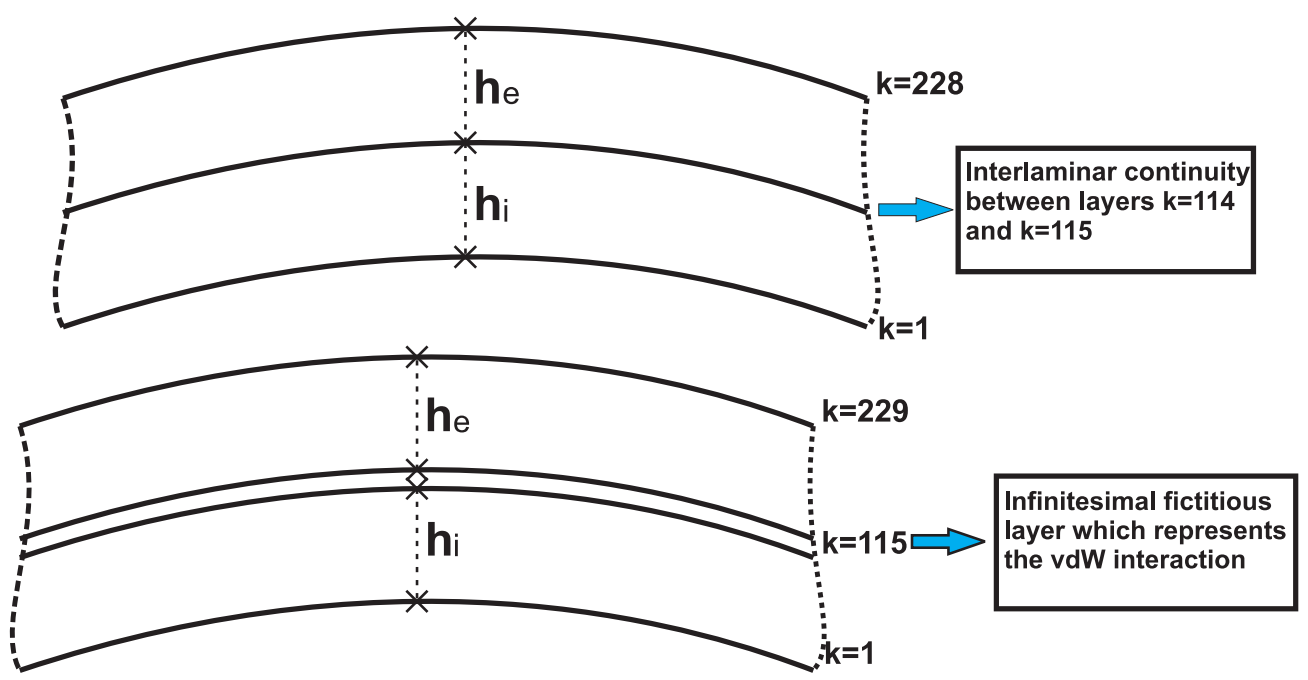

Figure 3. Continuum 3D shell model for DWCNT analysis. Interlaminar continuity between the two cylinders (at the top) and van der Waals interaction between the two cylinders (at the bottom).

Table 6. Second benchmark, simply supported double-walled carbon nanotubes (DWCNT) (first geometry with $R_{\alpha} / h=0.75$ ). Frequencies in GHz for 2D GDQ and 3D exact shell models.

\begin{tabular}{|c|c|c|c|c|}
\hline \multicolumn{5}{|c|}{ Nanotube 1} \\
\hline Mode $(\mathbf{p}, \mathbf{q})$ & 3D Exact & 3D $\mathrm{vdW}_{\mathrm{w}}$ Exact & ESL-GDQ & LW-GDQ \\
\hline \multicolumn{5}{|c|}{$L / d_{e}=5$} \\
\hline $\mathrm{I}(2,1)$ & 277.4 & 270.8 & 277.4 & 277.4 \\
\hline I $(2,2)$ & 946.2 & 892.3 & 946.2 & 946.2 \\
\hline $\mathrm{I}(2,3)$ & 1785 & 1628 & 1785 & 1785 \\
\hline \multicolumn{5}{|c|}{$L / d_{e}=10$} \\
\hline $\mathrm{I}(2,1)$ & 73.12 & 72.64 & 73.12 & 73.12 \\
\hline $\mathrm{I}(2,2)$ & 277.4 & 270.8 & 277.4 & 277.4 \\
\hline $\mathrm{I}(2,3)$ & 579.4 & 554.3 & 579.4 & 579.4 \\
\hline \multicolumn{5}{|c|}{$L / d_{e}=15$} \\
\hline $\mathrm{I}(2,1)$ & 32.85 & 32.76 & 32.85 & 32.85 \\
\hline $\mathrm{I}(2,2)$ & 128.1 & 126.6 & 128.1 & 128.1 \\
\hline $\mathrm{I}(2,3)$ & 277.4 & 270.8 & 277.4 & 277.4 \\
\hline \multicolumn{5}{|c|}{$L / d_{e}=30$} \\
\hline $\mathrm{I}(2,1)$ & 8.266 & 8.266 & 8.266 & 8.266 \\
\hline $\mathrm{I}(2,2)$ & 32.85 & 32.76 & 32.85 & 32.85 \\
\hline $\mathrm{I}(2,3)$ & 73.12 & 72.64 & 73.12 & 73.12 \\
\hline \multicolumn{5}{|c|}{$L / d_{e}=50$} \\
\hline $\mathrm{I}(2,1)$ & 2.980 & 2.980 & 2.980 & 2.980 \\
\hline $\mathrm{I}(2,2)$ & 11.89 & 11.89 & 11.89 & 11.89 \\
\hline $\mathrm{I}(2,3)$ & 26.65 & 26.60 & 26.65 & 26.65 \\
\hline
\end{tabular}



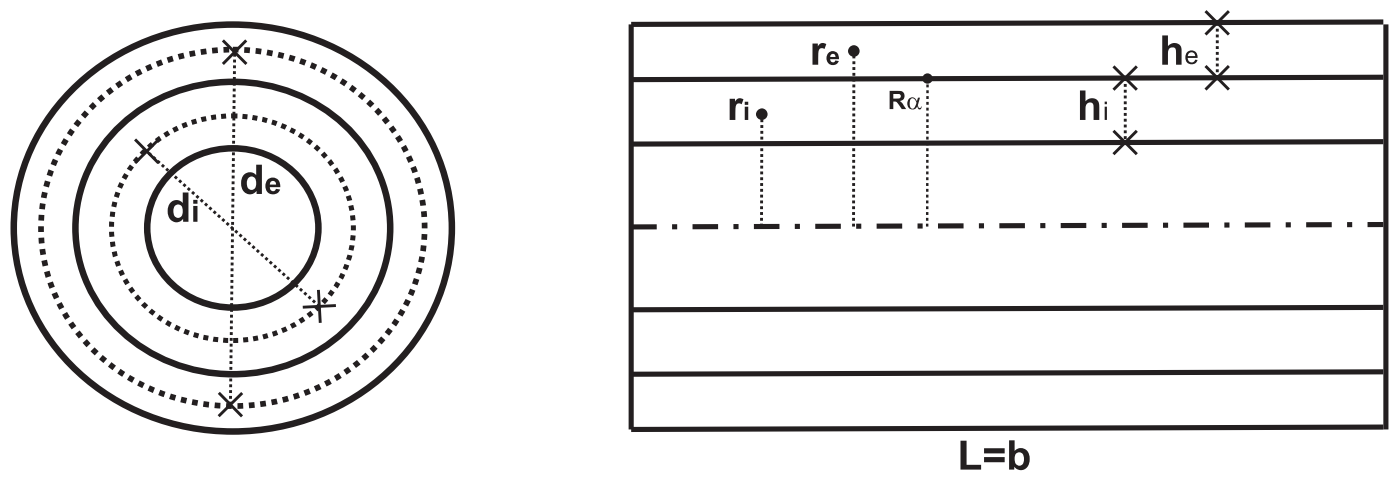

Figure 4. Geometrical data for the equivalent continuum DWCNT analyzed in the benchmark.
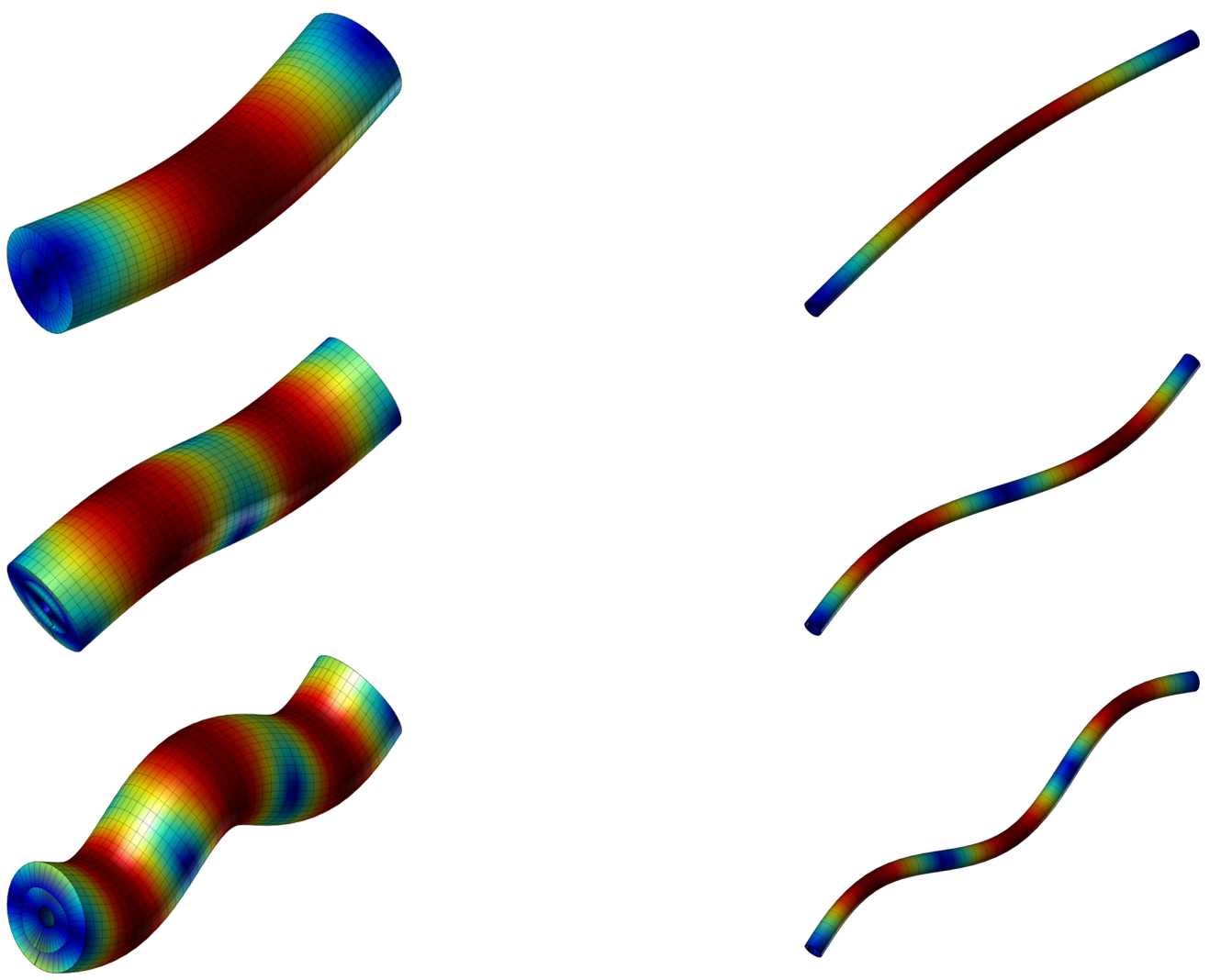

Figure 5. DWCNT benchmark: first geometry for $L / d_{e}=5$ on the left side and fourth geometry for $L / d_{e}=30$ on the right side. Vibration modes via the LW-GDQ model for half-wave numbers $p=2$ and $q=1,2,3$. 
Table 7. Second benchmark, simply supported DWCNT (second geometry with $R_{\alpha} / h=2.36$ ). Frequencies in GHz for 2D GDQ and 3D exact shell models.

\begin{tabular}{|c|c|c|c|c|}
\hline \multicolumn{5}{|c|}{ Nanotube 2} \\
\hline Mode $(\mathbf{p}, \mathbf{q})$ & 3D Exact & 3D $\mathrm{vdW}_{\mathrm{v}}$ Exact & ESL-GDQ & LW-GDQ \\
\hline \multicolumn{5}{|c|}{$L / d_{e}=5$} \\
\hline $\mathrm{I}(2,1)$ & 106.5 & 106.2 & 106.5 & 106.5 \\
\hline $\mathrm{I}(2,2)$ & 347.7 & 346.1 & 347.7 & 347.7 \\
\hline $\mathrm{I}(2,3)$ & 629.9 & 624.2 & 629.9 & 629.9 \\
\hline \multicolumn{5}{|c|}{$L / d_{e}=10$} \\
\hline $\mathrm{I}(2,1)$ & 28.55 & 28.54 & 28.55 & 28.55 \\
\hline I $(2,2)$ & 106.5 & 106.2 & 106.5 & 106.5 \\
\hline $\mathrm{I}(2,3)$ & 217.6 & 216.1 & 217.6 & 217.6 \\
\hline \multicolumn{5}{|c|}{$L / d_{e}=15$} \\
\hline $\mathrm{I}(2,1)$ & 12.88 & 12.87 & 12.88 & 12.88 \\
\hline $\mathrm{I}(2,2)$ & 49.79 & 49.74 & 49.79 & 49.79 \\
\hline I $(2,3)$ & 106.5 & 106.2 & 106.5 & 106.5 \\
\hline \multicolumn{5}{|c|}{$L / d_{e}=30$} \\
\hline $\mathrm{I}(2,1)$ & 3.248 & 3.248 & 3.248 & 3.248 \\
\hline I $(2,2)$ & 12.88 & 12.87 & 12.88 & 12.88 \\
\hline $\mathrm{I}(2,3)$ & 28.55 & 28.53 & 28.55 & 28.55 \\
\hline \multicolumn{5}{|c|}{$L / d_{e}=50$} \\
\hline $\mathrm{I}(2,1)$ & 1.172 & 1.171 & 1.172 & 1.172 \\
\hline $\mathrm{I}(2,2)$ & 4.671 & 4.671 & 4.671 & 4.671 \\
\hline $\mathrm{I}(2,3)$ & 10.45 & 10.45 & 10.45 & 10.45 \\
\hline
\end{tabular}

Table 8. Second benchmark, simply supported DWCNT (third geometry with $R_{\alpha} / h=3.68$ ). Frequencies in GHz for 2D GDQ and 3D exact shell models.

\begin{tabular}{|c|c|c|c|c|}
\hline \multicolumn{5}{|c|}{ Nanotube 3} \\
\hline $\operatorname{mode}(p, q)$ & 3D Exact & $3 D_{v d W}$ Exact & ESL-GDQ & LW-GDQ \\
\hline \multicolumn{5}{|c|}{$L / d_{e}=5$} \\
\hline $\mathrm{I}(2,1)$ & 71.74 & 71.70 & 71.74 & 71.74 \\
\hline $\mathrm{I}(2,2)$ & 231.5 & 231.3 & 231.5 & 231.5 \\
\hline $\mathrm{I}(2,3)$ & 414.6 & 414.4 & 414.6 & 414.6 \\
\hline \multicolumn{5}{|c|}{$L / d_{e}=10$} \\
\hline $\mathrm{I}(2,1)$ & 19.34 & 19.33 & 19.34 & 19.34 \\
\hline $\mathrm{I}(2,2)$ & 71.74 & 71.70 & 71.74 & 71.74 \\
\hline $\mathrm{I}(2,3)$ & 145.8 & 145.6 & 145.8 & 145.8 \\
\hline \multicolumn{5}{|c|}{$L / d_{e}=15$} \\
\hline $\mathrm{I}(2,1)$ & 8.729 & 8.729 & 8.729 & 8.729 \\
\hline $\mathrm{I}(2,2)$ & 33.67 & 33.66 & 33.67 & 33.67 \\
\hline $\mathrm{I}(2,3)$ & 71.74 & 71.70 & 71.74 & 71.74 \\
\hline \multicolumn{5}{|c|}{$L / d_{e}=30$} \\
\hline $\mathrm{I}(2,1)$ & 2.203 & 2.203 & 2.203 & 2.203 \\
\hline $\mathrm{I}(2,2)$ & 8.729 & 8.729 & 8.729 & 8.729 \\
\hline I $(2,3)$ & 19.34 & 19.33 & 19.34 & 19.34 \\
\hline \multicolumn{5}{|c|}{$L / d_{e}=50$} \\
\hline $\mathrm{I}(2,1)$ & 0.7949 & 0.7946 & 0.7949 & 0.7949 \\
\hline $\mathrm{I}(2,2)$ & 3.168 & 3.168 & 3.168 & 3.168 \\
\hline $\mathrm{I}(2,3)$ & 7.088 & 7.087 & 7.088 & 7.088 \\
\hline
\end{tabular}


Table 9. Second benchmark, simply supported DWCNT (fourth geometry with $R_{\alpha} / h=5.25$ ).

Frequencies in GHz for 2D GDQ and 3D exact shell models.

\begin{tabular}{|c|c|c|c|c|}
\hline \multicolumn{5}{|c|}{ Nanotube 4} \\
\hline mode $(p, q)$ & 3D exact & $3 \mathrm{D}_{v d W}$ exact & ESL-GDQ & LW-GDQ \\
\hline \multicolumn{5}{|c|}{$L / d_{e}=5$} \\
\hline $\mathrm{I}(2,1)$ & 51.79 & 51.78 & 51.79 & 51.79 \\
\hline $\mathrm{I}(2,2)$ & 166.1 & 166.1 & 166.1 & 166.1 \\
\hline $\mathrm{I}(2,3)$ & 295.6 & 295.6 & 295.6 & 295.6 \\
\hline \multicolumn{5}{|c|}{$L / d_{e}=10$} \\
\hline $\mathrm{I}(2,1)$ & 14.00 & 14.00 & 14.00 & 14.00 \\
\hline $\mathrm{I}(2,2)$ & 51.79 & 51.78 & 51.79 & 51.79 \\
\hline $\mathrm{I}(2,3)$ & 104.9 & 104.9 & 104.9 & 104.9 \\
\hline \multicolumn{5}{|c|}{$L / d_{e}=15$} \\
\hline $\mathrm{I}(2,1)$ & 6.323 & 6.323 & 6.323 & 6.323 \\
\hline $\mathrm{I}(2,2)$ & 24.35 & 24.35 & 24.35 & 24.35 \\
\hline $\mathrm{I}(2,3)$ & 51.79 & 51.78 & 51.79 & 51.79 \\
\hline \multicolumn{5}{|c|}{$L / d_{e}=30$} \\
\hline $\mathrm{I}(2,1)$ & 1.597 & 1.597 & 1.597 & 1.597 \\
\hline $\mathrm{I}(2,2)$ & 6.323 & 6.323 & 6.323 & 6.323 \\
\hline $\mathrm{I}(2,3)$ & 14.00 & 14.00 & 14.00 & 14.00 \\
\hline \multicolumn{5}{|c|}{$L / d_{e}=50$} \\
\hline $\mathrm{I}(2,1)$ & 0.5761 & 0.5761 & 0.5761 & 0.5761 \\
\hline $\mathrm{I}(2,2)$ & 2.296 & 2.296 & 2.296 & 2.296 \\
\hline $\mathrm{I}(2,3)$ & 5.135 & 5.135 & 5.135 & 5.135 \\
\hline
\end{tabular}

\section{Conclusions}

The paper proposed the free vibration analysis of simply supported SWCNTs and DWCNTs. A 3D exact model and several 2D GDQ models have been used for such an analysis. Two-dimensional GDQ models are the classical Reissner-Mindlin approach (RM-GDQ) and two refined fourth order models (an equivalent single layer approach (ESL-GDQ) and a layer wise approach (LW-GDQ)). The 3D exact model is a layer wise approach, and it uses the interlaminar continuity conditions between the two cylinders which simulate the DWCNT. A second 3D exact model (3D $\mathrm{D}_{v d W}$ exact model) has also been considered. In this case, the two isotropic cylinders, which represent the DWCNT, are linked by means of the van der Waals forces. The van der Waals forces are indicated as an opportune fictitious layer between the two cylinders. Comparisons in terms of frequencies between the $3 \mathrm{D}$ exact and the $3 \mathrm{D}_{v d W}$ exact model allow one to understand the van der Waals effects. These effects are very small (in general, they can be neglected), but they become important for short DWCNTs, small "radius of curvature/thickness" ratios and/or higher frequencies. ESL-GDQ and LW-GDQ models always give the same results in terms of frequency for DWCNTs because these structures are made of two isotropic cylinders (without any transverse anisotropy). In this case, an equivalent single layer approach is coincident with a layer wise approach (for the same order of expansion used for the displacement components). For SWCNT analysis, the use of beam models is inappropriate for short CNTs. In this case, refined 2D or 3D shell models are mandatory (in particular, in the case of small "radius of curvature/thickness" ratios) for a correct frequency evaluation. 


\section{Author Contributions}

The authors contributed equally to this work. All authors have read and approved the final manuscript.

\section{Conflicts of Interest}

The authors declare no conflict of interest.

\section{References}

1. Valavala, P.K.; Odegard, G.M. Modeling techniques for determination of mechanical properties of polymer nanocomposites. Rev. Adv. Mater. Sci. 2005, 9, 34-44.

2. Rouainia, G.; Djeghaba, K. Evaluation of Young's modulus of single walled carbon nanotube (SWNT) reinforced concrete composite. J. Eng. Appl. Sci. 2008, 3, 504-515.

3. Iijima, S. Helical microtubules of graphitic carbon. Nature 1991, 354, 56-58.

4. Wang, C.Y.; Zhang, L.C. A critical assessment of the elastic properties and effective wall thickness of single-walled carbon nanotubes. Nanotechnology 2008, 19, 1-5.

5. Qian, D.; Wagner, G.J.; Liu, W.K.; Yu, M.F.; Ruoff, R.S. Mechanics of carbon nanotubes. Appl. Mech. Rev. 2002, 55, 495-533.

6. Chen, X.; Cao, G. A structural mechanics study of single-walled carbon nanotubes generalized from atomistic simulation. Nanotechnology 2006, 17, 1004-1015.

7. Hu, Y.G.; Liew, K.M.; Wang, Q. Modeling of vibrations of carbon nanotubes. Procedia Eng. 2012, 31, 343-347.

8. Ansari, R.; Ajori, S.; Arash, B. Vibrations of single- and double-walled carbon nanotubes with layerwise boundary conditions: A molecular dynamics study. Curr. Appl. Phys. 2012, 12, 707-711.

9. Chowdhury, R.; Adhikari, S.; Wang, C.Y.; Scarpa, F. A molecular mechanics approach for the vibration of single-walled carbon nanotubes. Comput. Mater. Sci. 2010, 48, 730-735.

10. Das, S.L.; Mandal, T.; Gupta, S.S. Inextensional vibration of zig-zag single-walled carbon nanotubes using nonlocal elasticity theories. Int. J. Solids Struct. 2013, 50, 2792-2797.

11. Zhang, Y.Y.; Wang, C.M.; Tan, V.B.C. Assessment of Timoshenko beam models for vibrational behavior of single-walled carbon nanotubes using molecular dynamics. Adv. Appl. Math. Mech. 2009, 1, 89-106.

12. Sinnott, S.S.; Mao, Z.; Lee, K.-H. Computational studies of molecular diffusion through carbon nanotube based membranes. Comput. Model. Eng. Sci. 2002, 3, 589-600.

13. Brenner, D.W.; Shenderova, O.A.; Areshkin, D.A.; Schall, J.D.; Frankland, S.-J.V. Atomic modeling of carbon-based nanostructures as a tool for developing new materials and technologies. Comput. Model. Eng. Sci. 2002, 3, 643-674.

14. Yang, L.; Han, J.; Anantram, M.P.; Jaffe, R.L. Bonding geometry and bandgap changes of carbon nanotubes under uniaxial and torsional strain. Comput. Model. Eng. Sci. 2002, 3, 675-686.

15. Namilae, S.; Chandra, U.; Srinivasan, A.; Chandra, N. Effect of interface modification on the mechanical behavior of carbon nanotube reinforced composites using parallel molecular dynamics simulations. Comput. Model. Eng. Sci. 2007, 22, 189-202. 
16. Arghavan, S.; Singh, A.V. On the vibrations of single-walled carbon nanotubes. J Sound Vib. 2011, 330, 3102-3122.

17. Gupta, A.; Sharma, S.C.; Harsha, S.P. Vibration analysis of carbon nanotubes based mass sensor using different boundary conditions. Int. J. Mech. Sci. 2012, 2, 8-12.

18. Mir, M.; Hosseini, A.; Majzoobi, G.H. A numerical study of vibrational properties of single-walled carbon nanotubes. Comput. Mater. Sci. 2008, 43, 540-548.

19. Yan, Y.; Shi, G.; Zhao, P. Frequency study of single-walled carbon nanotubes based on a space-frame model with flexible connections. J. Comput. 2011, 6, 1125-1130.

20. Aydogdu, M. Axial vibration of the nanorods with the non local continuum rod model. Phys. E 2009, 41, 861-864.

21. Yan, J.W.; Liew, K.M.; He, L.H. Free vibration analysis of single-walled carbon nanotubes using a higher-order gradient theory. J Sound Vib. 2013, 332, 3740-3755.

22. Wang, C.Y.; Zhang L.C. A critical assessment of the elastic properties and effective wall thickness of single-walled carbon nanotubes. Nanotechnology 2008, 19, 1-5.

23. Wang, C.Y.; Zhang L.C. An elastic shell model for characterizing single-walled carbon nanotubes. Nanotechnology 2008, 19, 1-6.

24. Vodenitcharova, T.; Zhang, L.C. Effective wall thickness of a single-walled carbon nanotube. Phys. Rev. B 2003, 68, 1-4.

25. Odegard, G.M.; Gates, T.S.; Nicholson, L.M.; Wise, K.E. Equivalent-continuum modeling of nano-structured materials. Compos. Sci. Technol. 2002, 62, 1869-1880.

26. Lee, U.; Oh, H. Evaluation of the structural properties of single-walled carbon nanotubes using a dynamic continuum modeling method. Mech. Adv. Mater. Struct. 2008, 15, 79-87.

27. Zhang, L.C. On the mechanics of single-walled carbon nanotubes. J. Mater. Process. Technol. 2009, 209, 4223-4228.

28. Araújo dos Santos, J.V. Effective elastic moduli evaluation of single walled carbon nanotubes using flexural vibrations. Mech. Adv. Mater. Struct. 2011, 18, 262-271.

29. Azrar, A.; Azrar, L.; Aljinaidi, A.A. Length scale effect analysis on vibration behavior of single walled carbon nanotubes with arbitrary boundary conditions. Rev. Mec. Appl. Theor. 2011, 2, $475-485$.

30. Benzair, A.; Tounsi, A.; Besseghier, A.; Heireche, H.; Moulay, N.; Boumia, L. The thermal effect on vibration of single-walled carbon nanotubes using nonlocal Timoshenko beam theory. J. Phys. D Appl. Phys. 2011, 41, 1-10.

31. Demir, C.; Civalek, O.; Akgöz, B. Free vibration analysis of carbon nanotubes based on shear deformable beam theory by discrete singular convolution technique. Math. Comput. Appl. 2010, 15, 57-65.

32. Foda, M.A. Steady state vibration analysis and mitigation of single-walled carbon nanotubes based on nonlocal Timoshenko beam theory. Comput. Mater. Sci. 2013, 71, 38-46.

33. Horng, T.L. Transverse vibration analysis of single-walled carbon nanotubes embedded in an elastic medium using Bernoulli-Fourier method. J. Surf. Eng. Mater. Adv. Technol. 2012, 2, 203-209. 
34. Ming, L.; Huiming, Z. Small scale effect on thermal vibration of single-walled carbon nanotubes with nonlocal boundary condition. Res. J. Appl. Sci. Eng. Technol. 2013, 5, 2729-2733.

35. Swain, A.; Roy, T.; Nanda, P.K. Vibration behaviour of single walled carbon nanotube using finite element. Int. J. Theor. Appl. Res. Mech. Eng. 2013, 2, 129-133.

36. Wang, B.; Deng, Z.C.; Zhang, K. Nonlinear vibration of embedded single-walled carbon nanotube with geometrical imperfection under harmonic load based on nonlocal Timoshenko beam theory. Appl. Math. Mech. 2013, 34, 269-280.

37. Wang, B.L.; Wang, K.F. Vibration analysis of embedded nanotubes using nonlocal continuum theory. Compos. B Eng. 2013, 47, 96-101.

38. Soltani, P.; Kassaei, A.; Taherian, M.M.; Farshidianfar, A. Vibration of wavy single-walled carbon nanotubes based on nonlocal Euler-Bernoulli and Timoshenko models. Int. J. Adv. Struct. Eng. 2012, 4, 1-10.

39. Simsek, M. Vibration analysis of a single-walled carbon nanotube under action of a moving harmonic load based on nonlocal elasticity theory. Phys. E 2010, 43, 182-191.

40. Murmu, T.; Pradhan, S.C. Buckling analysis of a single-walled carbon nanotube embedded in an elastic medium based on nonlocal elasticity and Timoshenko beam theory and using DQM. Phys. E 2009, 41, 1232-1239.

41. Aydogdu, M. A general non local beam theory: Its application to nanobeam bending, buckling and vibration. Phys. E 2009, 41, 1651-1655.

42. Aydogdu, M. Vibration of multi-walled carbon nanotubes by generalized shear deformation theory. Int. J. Mech. Sci. 2008, 50, 837-844.

43. Khosrozadeh, A.; Hajabasi, M.A. Free vibration of embedded double-walled carbon nanotubes considering nonlinear interlayer van der Waals forces. Appl. Math. Model. 2012, 36, 997-1007.

44. Fang, B.; Zhen, Y.-X.; Zhang, C.-P.; Tang, Y. Nonlinear vibration analysis of double-walled carbon nanotubes based on nonlocal elasticity theory. Appl. Math. Model. 2013, 37, 1096-1107.

45. Natsuki, T.; Ni, Q.-Q.; Endo, M. Analysis of the vibration characteristics of double-walled carbon nanotubes. Carbon 2008, 46, 1570-1573.

46. Kiani, K. Vibration analysis of elastically restrained double-walled carbon nanotubes on elastic foundation subjected to axial load using nonlocal shear deformable beam theories. Int. J. Mech. Sci. 2013, 68, 16-34.

47. Aydogdu, M. Longitudinal wave propagation in multiwalled carbon nanotubes. Compos. Struct. 2014, 107, 578-584.

48. de Borbón, F.; Ambrosini, D. On the influence of van der Waals coefficient on the transverse vibration of double walled carbon nanotubes. Comput. Mater. Sci. 2012, 65, 504-508.

49. Chang, T.-P. Stochastic FEM on nonlinear vibration of fluid-loaded double-walled carbon nanotubes subjected to a moving load based on nonlocal elasticity theory. Compos. B Eng. 2013, 54, 391-399.

50. Benguediab, S.; Tounsi, A.; Zidour, M.; Semmah, A. Chirality and scale effects on mechanical buckling properties of zigzag double-walled carbon nanotubes. Compos. B Eng. 2014, 57, 21-24.

51. Brischetto, S. A continuum elastic three-dimensional model for natural frequencies of single-walled carbon nanotubes. Compos. B Eng. 2014, 61, 222-228. 
52. Wang, C.Y.; Zhang, L.C. An elastic shell model for characterizing single-walled carbon nanotubes. Nanotechnology 2008, 19, 1-6.

53. Alibeigloo, A.; Shaban, M. Free vibration analysis of carbon nanotubes by using three-dimensional theory of elasticity. Acta Mech. 2013, 224, 1415-1427.

54. Mikhasev, M. On localized modes of free vibrations of single-walled carbon nanotubes embedded in nonhomogeneous elastic medium. Z. Angew. Math. Mech. 2013, 1-12.

55. Silvestre, N.; Wang, C.M.; Zhang, Y.Y.; Xiang, Y. Sanders shell model for buckling of single-walled carbon nanotubes with small aspect ratio. Compos. Struct. 2011, 93, 1683-1691.

56. Strozzi, M.; Manevitch, L.I.; Pellicano, F.; Smirnov, V.V.; Shepelev, D.S. Low-frequency linear vibrations of single-walled carbon nanotubes: Analytical and numerical models. J Sound Vib. 2014, 333, 2936-2957.

57. Liew, K.M.; Wang, Q. Analysis of wave propagation in carbon nanotubes via elastic shell theories. Int. J. Eng. Sci. 2007, 45, 227-241.

58. Cinefra, M.; Carrera, E.; Brischetto, S. Refined shell models for the vibration analysis of multiwalled carbon nanotubes. Mech. Adv. Mater. Struct. 2011, 18, 476-483.

59. Brischetto, S. A Continuum shell model including van derWaals interaction for free vibrations of double-walled carbon nanotubes. Comput. Model. Eng. Sci. 2015, 104, 305-327.

60. Dong, K.; Zhu, S.Q.; Wang, X. Wave propagation in multiwall carbon nanotubes embedded in a matrix material. Compos. Struct. 2008, 82, 1-9.

61. Li, R.; Kardomateas, G.A. Vibration characteristics of multiwalled carbon nanotubes embedded in elastic media by a nonlocal elastic shell model. J. Appl. Mech. 2007, 74, 1087-1094.

62. Yao, X., Han, Q. Torsional buckling and postbuckling equilibrium path of double-walled carbon nanotubes. Compos. Sci. Technol. 2008, 68, 113-120.

63. He, X.Q.; Kitipornchai, S.; Wang, C.M.; Liew, K.M. Modeling of van der Waals force for infinitesimal deformation of multi-walled carbon nanotubes treated as cylindrical shells. Int. J. Solids Struct. 2005, 42, 6032-6047.

64. Han, Q.; Lu, G.; Dai, L. Bending instability of an embedded double-walled carbon nanotube based on Winkler and van der Waals models. Compos. Sci. Technol. 2005, 65, 1337-1346.

65. Hoseinzadeh, M.S.; Khadem, S.E. Thermoelastic vibration and damping analysis of double-walled carbon nanotubes based on shell theory. Phys. E 2011, 43, 1146-1154.

66. Brischetto, S. Exact elasticity solution for natural frequencies of functionally graded simply-supported structures. Comput. Model. Eng. 2013, 95, 391-430.

67. Brischetto, S. An exact 3D solution for free vibrations of multilayered cross-ply composite and sandwich plates and shells. Int. J. Appl. Mech. 2014, 6, 1-42.

68. Brischetto, S. Three-dimensional exact free vibration analysis of spherical, cylindrical, and flat one-layered panels. Shock Vib. 2014, 2014, 1-29.

69. Brischetto, S.; Torre, R. Exact 3D solutions and finite element 2D models for free vibration analysis of plates and cylinders. Curved Layer. Struct. 2014, 1, 59-92.

70. Messina, A. Three Dimensional Free Vibration Analysis of Cross-Ply Laminated Plates through 2D and Exact Models. In Proceedings of the 3rd International Conference on Integrity, Reliability and Failure, Porto, Portugal, 20-24 July 2009. 
71. Soldatos, K.P.; Ye, J. Axisymmetric static and dynamic analysis of laminated hollow cylinders composed of monoclinic elastic layers. J Sound Vib. 1995, 184, 245-259.

72. Tornabene, F.; Viola, E.; Fantuzzi, N. General higher-order equivalent single layer theory for free vibrations of doubly-curved laminated composite shells and panels. Compos. Struct. 2013, 104, 94-117.

73. Tornabene, F. General higher order layer-wise theory for free vibrations of doubly-curved laminated composite shells and panels. Mech. Adv. Mater. Struct. 2015, in press.

74. Tornabene, F.; Fantuzzi, N.; Bacciocchi, M. Free vibrations of free-form doubly-curved shells made of functionally graded materials using higher-order equivalent single layer theories. Compos. B Eng. 2014, 67, 490-509.

75. Tornabene, F.; Fantuzzi, N.; Bacciocchi, M. The local GDQ method applied to general higher-order theories of doubly-curved laminated composite shells and panels: The free vibration analysis. Compos. Struct. 2014, 116, 637-660.

76. Tornabene, F.; Fantuzzi, N.; Viola E.; Reddy, J.N. Winkler-Pasternak foundation effect on the static and dynamic analyses of laminated doubly-curved and degenerate shells and panels. Compos. B Eng. 2014, 57, 269-296.

77. Tornabene, F.; Fantuzzi, N.; Viola E.; Ferreira, A.J.M. Radial basis function method applied to doubly-curved laminated composite shells and panels with a general higher-order equivalent single layer theory. Compos. B Eng. 2013, 55, 642-659.

78. Viola, E.; Tornabene, F.; Fantuzzi, N. General higher-order shear deformation theories for the free vibration analysis of completely doubly-curved laminated shells and panels. Compos. Struct. 2013, 95, 639-666.

79. Tornabene, F.; Fantuzzi, N.; Bacciocchi, M.; Viola, E. Accurate inter-laminar recovery for plates and doubly-curved shells with variable radii of curvature using layer-wise theories. Compos. Struct. 2015, 124, 368-393.

80. Tornabene, F.; Fantuzzi, N.; Ubertini, F.; Viola, E. Strong formulation finite element method based on differential quadrature: A survey. Appl. Mech. Rev. 2015, 67, doi:10.1115/1.4028859.

81. Tornabene, F.; Brischetto, S.; Fantuzzi, N.; Viola, E. Numerical and exact models for free vibration analysis of cylindrical and spherical shell panels. Compos. B Eng. 2015, 81, 231-250.

82. Carrera, E.; Brischetto, S.; Nali, P. Plates and Shells for Smart Structures: Classical and Advanced Theories for Modeling and Analysis; John Wiley \& Sons, Ltd.: New Delhi, India, 2011.

(c) 2015 by the authors; licensee MDPI, Basel, Switzerland. This article is an open access article distributed under the terms and conditions of the Creative Commons Attribution license (http://creativecommons.org/licenses/by/4.0/). 